\title{
Neuroprotective Effects of GV1001 in Animal Stroke Model and Neural Cells Subject to Oxygen- Glucose Deprivation/Reperfusion Injury
}

\author{
Hyuk Sung Kwon, ${ }^{\mathrm{a}, *}$ Ye Eun Kim, ${ }^{\mathrm{a}, *}$ Hyun-Hee Park, ${ }^{\mathrm{a}, *}$ Jeong-Woo Son, ${ }^{\mathrm{a}}$ Hojin Choi, ${ }^{\mathrm{a}}$ Young Joo Lee, ${ }^{\mathrm{a}}$ \\ Hyun Young Kim, ${ }^{\mathrm{b}}$ Kyu-Yong Lee, ${ }^{\mathrm{a}}$ Seong-Ho Koh ${ }^{\mathrm{a}, \mathrm{c}}$ \\ aDepartment of Neurology, Hanyang University Guri Hospital, Hangyang University College of Medicine, Guri, Korea \\ ${ }^{b}$ Department of Neurology, Hanyang University Seoul Hospital, Hanyang University College of Medicine, Seoul, Korea \\ 'Department of Translational Medicine, Hanyang University Graduate School of Biomedical Science \& Engineering, Seoul, Korea
}

Background and Purpose Previous studies have revealed the diverse neuroprotective effects of GV1001. In this study, we investigated the effects of GV1001 on focal cerebral ischemia-reperfusion injury (IRI) in rats and oxygen-glucose deprivation/reoxygenation (OGD/R)-induced injury in neural stem cells (NSCs) and cortical neurons.

Methods Focal cerebral IRI was induced by transient middle cerebral artery occlusion (MCAO). Brain diffusion-weighted imaging (DWI) was performed 2 hours after occlusion, and a total of 37 rats were treated by reperfusion with GV1001 or saline 2 hours after occlusion. Fluid-attenuated inversion recovery (FLAIR) magnetic resonance imaging, immunohistochemistry, and neurobehavioral function analyses were performed. Additionally, OGD/R-injured NSCs and cortical neurons were treated with different GV1001 concentrations. Cell viability, proliferation, migration, and oxidative stress were determined by diverse molecular analyses.

Results In the stroke model, GV1001 protected neural cells against IRI. The most effective dose of GV1001 was $60 \mu \mathrm{M} / \mathrm{kg}$. The infarct volume on FLAIR 48 hours after MCAO compared to lesion volume on DWI showed a significantly smaller ratio in the GV1001-treated group. GV1001-treated rats exhibited better behavioral functions than the saline-treated rats. Treatment with GV1001 increased the viability, proliferation, and migration of the OGD/R-injured NSCs. Free radicals were significantly restored by treatment with GV1001. These neuroprotective effects of GV1001 have also been demonstrated in 0GD/R-injured cortical neurons.

Conclusions The results suggest that GV1001 has neuroprotective effects against IRI in NSCs, cortical neurons, and the rat brain. These effects are mediated through the induction of cellular proliferation, mitochondrial stabilization, and anti-apoptotic, anti-aging, and antioxidant effects.
Correspondence: Seong-Ho Koh Department of Neurology, Hanyang University Guri Hospital, Hangyang University College of Medicine, 153 Gyeongchun-ro, Guri 11923, Korea Tel: +82-31-560-2267

Fax: +82-31-560-2267 E-mail: ksh213@hanyang.ac.kr https://orcid.org/0000-0001-5419-5761

Co-correspondence: Kyu-Yong Lee Department of Neurology, Hanyang University Guri Hospital, Hangyang University College of Medicine, 153 Gyeongchun-ro, Guri 11923, Korea Tel: +82-31-560-2266

Fax: +82-31-560-2267

E-mail: kylee@hanyang.ac.kr https://orcid.org/0000-0001-8855-7513

Received: February 15, 2021

Revised: June 14, 2021

Accepted: June 16, 2021

*These authors contributed equally to the manuscript as first author.

Keywords Ischemic stroke; GV1001 peptide; Neural stem cells; Models, animal

Copyright $\odot 2021$ Korean Stroke Society

This is an Open Access article distributed under the terms of the Creative Commons Attribution Non-Commercial License (http://creativecommons.org/licenses/by-nc/4.0/) which permits unrestricted non-commercial use, distribution, and reproduction in any medium, provided the original work is properly cited. 


\section{Introduction}

Acute cerebral ischemia elicits an immune response that leads to a cascade of events culminating in neuronal death and injury to supportive structures in the brain. ${ }^{1}$ Several mechanisms, including excitatory toxicity, oxidative stress, inflammation, and apoptosis, are thought to be involved in the pathogenesis of ischemic stroke. ${ }^{2}$ Additionally, neural stem cells (NSCs), which might contribute to the regeneration of the injured brain, can be damaged by ischemic injury. ${ }^{3}$ Substantial clinical trials with drugs that modulate these mechanisms have failed to show efficacy after ischemic stroke.

GV1001 is a vaccine peptide anticancer agent derived from a 16-amino-acid sequence in the active site of human telomerase reverse transcriptase (hTERT). hTERT has both telomeric and extratelomeric functions. ${ }^{4,5}$ By mimicking the extra-telomeric function of hTERT, GV1001 has various biological functions, including antioxidant, anti-inflammatory, and anti-apoptotic effects, induction of cell proliferation, and mitochondrial stabilization. These effects can protect against ischemia-reperfusion injury (IRI), ${ }^{3,6}$

Based on the diverse effects of GV1001, we hypothesized that it might have neuroprotective effects against injury due to cerebral ischemia and reperfusion. In this study, we investigated the protective effect of GV1001 against ischemic stroke in an animal model and oxygen-glucose deprivation/reoxygenation (OGD/R)-derived injury in NSCs.

\section{Methods}

\section{In vivo methods}

\section{Animal preparation and experimental protocols}

All animal procedures were performed in accordance with the Hanyang University Guidelines for the Care and Use of Laboratory Animals and were approved by the Institutional Animal Care and Use Committee of Hanyang University. Male Sprague-Dawley rats weighing 280 to $300 \mathrm{~g}$ were used (Biogenomics Incorporated, Seoul, Korea). In accordance with the Animal Research: Reporting of In Vivo Experiments (ARRIVE) guidelines, all experiments were conducted in a strictly blinded fashion using predetermined inclusion and exclusion criteria and attrition due to mortality and other causes reported. However, sample sizes a priori could not be calculated because anticipated differences in infarct size and estimated efficacy with GV1001 were not available.

\section{Middle cerebral artery occlusion of rats}

After a 7-day period of adaptation and pre-training for neurological examination, the left middle cerebral artery was oc- cluded for 2 hours and reperfused using the intraluminal filament technique described previously ${ }^{7,8}$ and in the Supplementary methods.

\section{Dose response and therapeutic time window of efficacy} In the first experiment to determine the most effective dose, rats were randomly divided into five groups: sham, saline, and different doses of GV1001 $(6,30$, and $60 \mu \mathrm{M} / \mathrm{kg})$-treated groups ( $n=10$ in each group). The sham group was subjected to the same protocol without middle cerebral artery occlusion (MCA0). Either saline or different doses of GV1001 (6, 30, and $60 \mu \mathrm{M} / \mathrm{kg}$ ) were subcutaneously injected immediately before reperfusion. All rats were sacrificed 48 hours after MCAO, and the infarct volume was calculated after staining with 2,3,5-triphenyltetrazolium chloride (Sigma-Aldrich, St. Louis, MO, USA) as described previously. Then, their brains were used for molecular biological assessment.

\section{Magnetic resonance imaging-based cohort}

After determining the optimal dose, another cohort randomized after diffusion-weighted imaging (DWI) was studied. The rats underwent DWI (repetition time [TR]/echo time $[T E]=4,455 / 74$ ms, slice thickness $/ \mathrm{gap}=1 / 1 \mathrm{~mm}$, matrix $=64 \times 63$, and field of view $[\mathrm{FOV}]=50 \times 50 \mathrm{~mm}$ ) at 3-Tesla using an Achieva device (Philips, Best, The Netherlands) 2 hours after MCAO. DWI lesion volumes were measured using the Medical Image Processing, Analysis, and Visualization (MIPAV) software (National Institutes of Health, Bethesda, MD, USA). Rats were divided blindly to receive either saline ( $n=13), 30 \mu \mathrm{M} / \mathrm{kg}$ GV1001 ( $n=12)$, or $60 \mu \mathrm{M} / \mathrm{kg}$ GV1001 $(n=12)$. Rats in the treatment and control groups were subjected to fluid-attenuated inversion recovery (FLAIR) magnetic resonance imaging (MRI) (TR/TE/inverstion time $[\mathrm{TI}]=11,000 / 77 / 2,800 \mathrm{~ms}$, slice thickness $/ \mathrm{gap}=1 / 1 \mathrm{~mm}$, matrix $=128 \times 128$, and $\mathrm{FOV}=50 \times 50$ $\mathrm{mm}) 48$ hours after MCAO. The final infarct volume was independently assessed with MIPAV by neurologists blinded to the experimental groups and was calculated from the average of the two measurements (Figure 1A).

Transferase-mediated deoxyuridine triphosphate nick end labeling assay

To detect apoptotic cells caused by ischemic stroke, a transferase-mediated deoxyuridine triphosphate nick end labeling (TUNEL) assay (Roche Boehringer Mannheim, Indianapolis, IN, USA) was performed. The sham group underwent the same protocol without MCAO, and the saline group was treated with saline. GV1001 was administered at doses of 30 and $60 \mu \mathrm{M} / \mathrm{kg}$. The detailed method for the TUNEL assay is described in the Supplementary methods. 


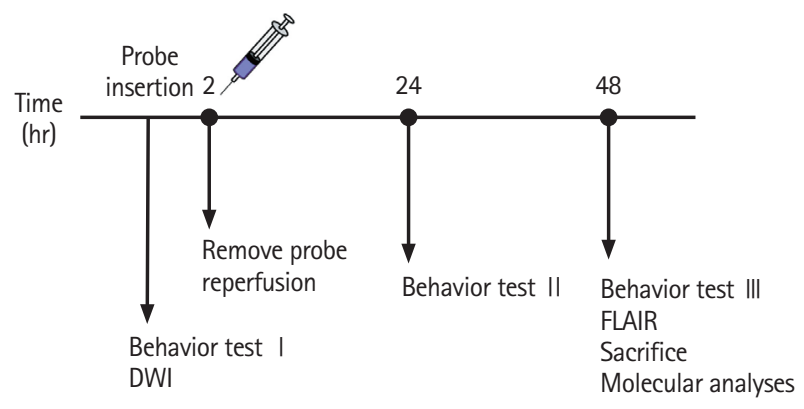

$$
\begin{array}{cl}
\text { SD-rat } & \cdot \text { Sham } \\
\text { 8 weeks } & \cdot 0.9 \% \text { Saline } \\
& \cdot 6 \mu \mathrm{M} / \mathrm{kg} \mathrm{GV1001} \\
& \cdot 30 \mu \mathrm{M} / \mathrm{kg} \mathrm{GV} 1001 \\
& \cdot 60 \mu \mathrm{M} / \mathrm{kg} \mathrm{GV} 1001 \\
& \rightarrow \text { Intravenous injection }
\end{array}
$$

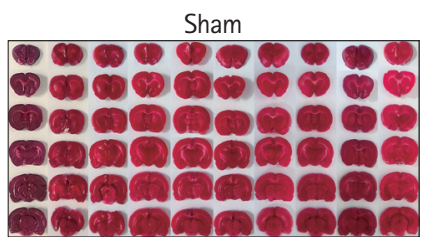

$6 \mu \mathrm{M} / \mathrm{kg} \mathrm{GV} 1001$

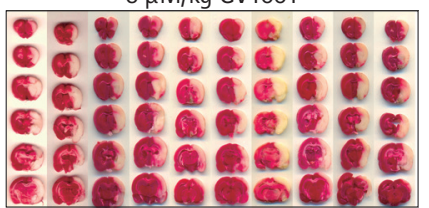

$60 \mu \mathrm{M} / \mathrm{kg} \mathrm{GV} 1001$

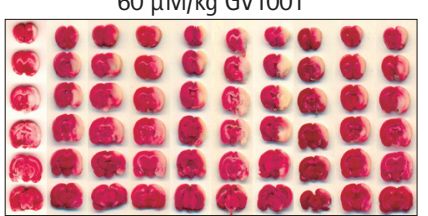

Infarct volume

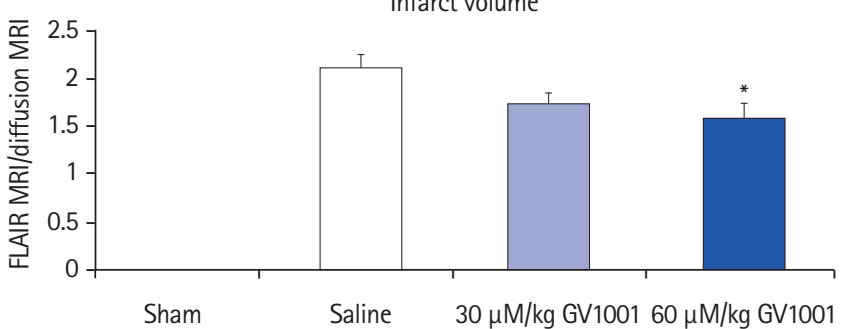

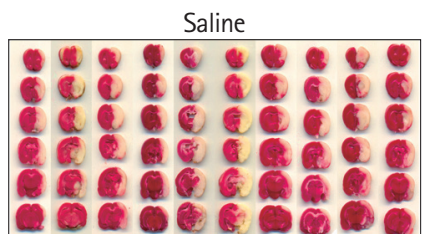

$30 \mu \mathrm{M} / \mathrm{kg} \mathrm{GV} 1001$
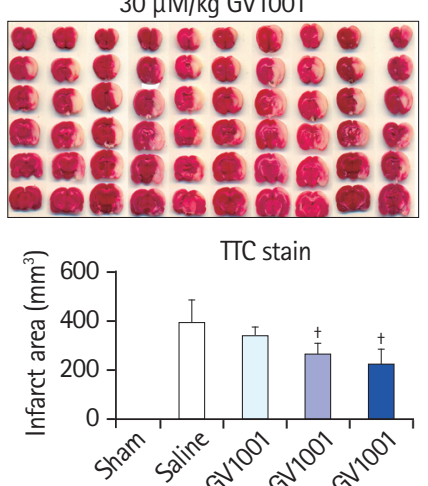

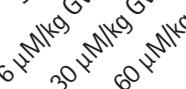
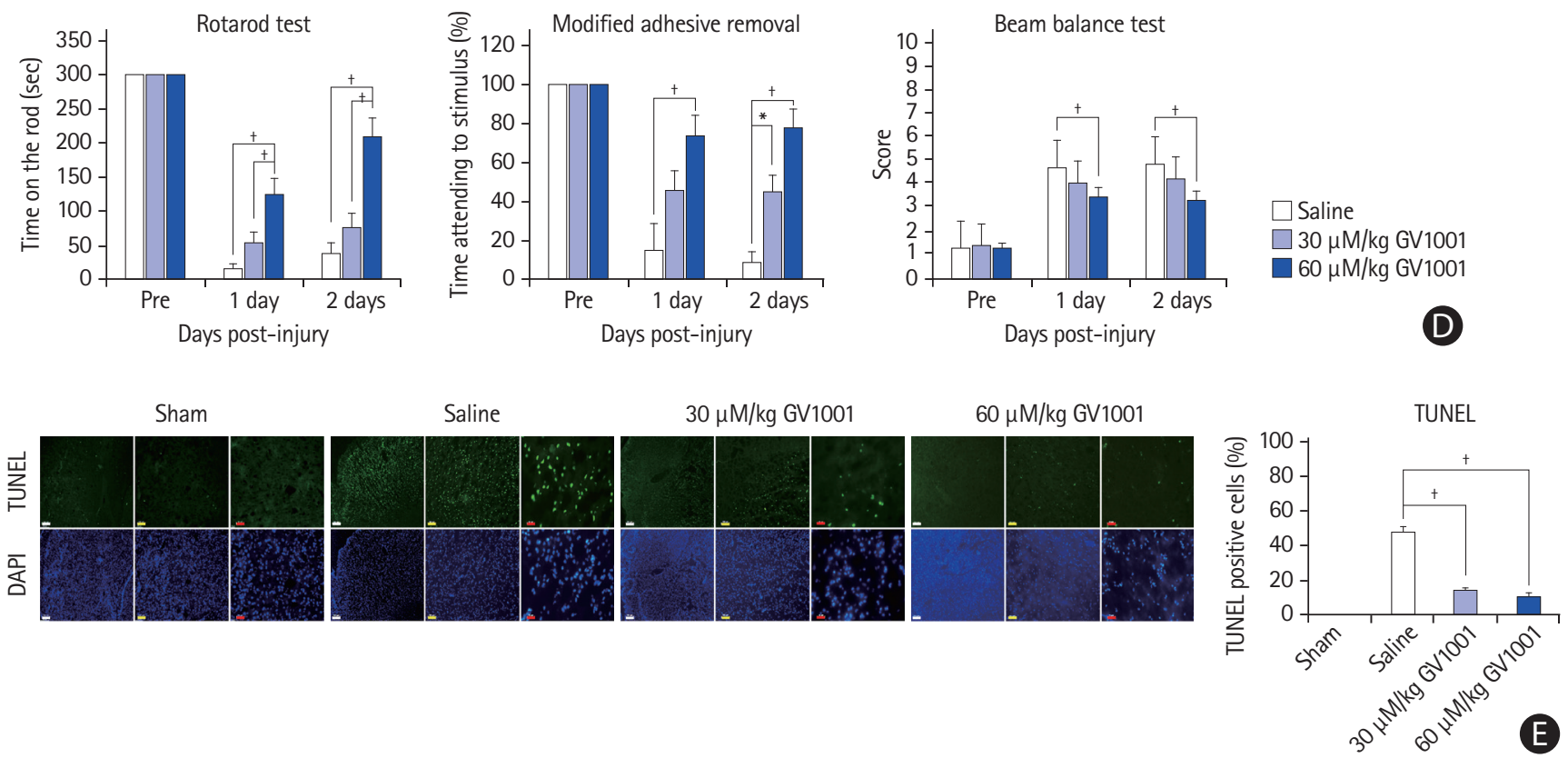

Figure 1. (A) Schematic representation of the study design and the timeline for the establishment of the ischemic stroke animal model. (B) 2,3,5-Triphenyltetrazolium chloride staining showing the amount of cerebral infarction in each group. The infarct volume was significantly reduced in groups treated with 30 and $60 \mu \mathrm{M} / \mathrm{kg}$ GV1001. (C) The infarct volume was measured by diffusion-weighted imaging (DWI) and fluid-attenuated inversion recovery (FLAIR) magnetic resonance imaging (MRI). The volume was significantly reduced in the $60 \mu \mathrm{M} / \mathrm{kg}$ GV1001 group. (D) All behavioral function tests showed a beneficial effect of GV1001. (E) The number of transferase-mediated deoxyuridine triphosphate nick end labeling (TUNEL)-positive cells was reduced in the GV1001-treated group, white scale bar: $250 \mu \mathrm{m}$; yellow scale bar: $100 \mu \mathrm{m}$; and red scale bar: $25 \mu \mathrm{m}$. SD, Sprague-Dawley; TTC, triphenyltetrazolium chloride; DAPI, 4',6-diamidino-2-phenylindole. ${ }^{*} P<0.05 ;{ }^{+} P<0.01$ (vs. control-saline group). 


\section{Neurological outcomes}

\section{1) Beam walking test}

The sensorimotor function was assessed using beam walking, rotarod, and modified sticky-tape tests performed before MCAO and 2 and 3 days after MCAO by an investigator blinded to the experimental groups. For the beam walking test, rats were trained 7 days before MCAO to walk on a wooden beam $(2.5 \times 2.5 \times 80 \mathrm{~cm})$ placed $60 \mathrm{~cm}$ above the floor to return to their home cage. The test was scored as follows: 0 , traverses the beam with no foot slip; 1, traverses by grasping the lateral side of the beam; 2 , shows disability walking on the beam but can traverse; 3 , takes a considerable amount of time to traverse the beam because of difficulty in walking; 4 , unable to traverse the beam; 5 , unable to move the body or any limb on the beam; 6 , unable to stay on the beam for 10 seconds. ${ }^{9}$

\section{2) Rotarod test}

Rats were placed on an accelerating rotarod cylinder, and the riding time that the animal remained on the rotarod was measured. The speed of the rotarod was not increased during the experiment. A trial ended if the animal fell off the rungs or gripped the device and spun around for two consecutive revolutions without attempting to walk on the rungs. The animals were trained 7 days before MCAO. Motor test data are presented as the mean duration (three trials) on the rotarod. ${ }^{9,10}$

\section{3) Modified sticky-tape test}

A sleeve was created using a $3 \mathrm{~cm}$ piece of green paper tape that was $1 \mathrm{~cm}$ wide. The tape was wrapped around the forepaw so that the tape attached to itself and allowed the fingers to protrude slightly from the sleeve that was formed. The typical response was for the rat to vigorously attempt to remove the sleeve by either pulling at the tape with its mouth and/or brushing the tape with its contralateral paw. After attaching the sleeve, the rat was placed in its cage and observed for 30 seconds. The timer was turned on only when the animal attempted to remove the taped sleeve. The data collected represented the fraction of the 30 seconds observation period that the animal spent attending to the stimulus. The contralateral and ipsilateral limbs were tested separately. The test was repeated three times per test day, and the best two scores were averaged. ${ }^{11}$

\section{Histology and immunohistochemistry}

All rats used for the evaluation of infarction volume with MRI were sacrificed 48 hours after MCAO for histological and immunohistochemical analyses. Hematoxylin and eosin (H\&E) staining distinguished the peri-infarct region from the remaining area. TUNEL-positive cells were counted in the GV1001 and control (sham and saline) groups. Immunohistochemical staining was performed as previously described ${ }^{7-9}$ using antibodies against phosphorylated Akt (pAkt; Ser473, 1:100, 9271, Cell Signaling Technology, Beverly, MA, USA), phospho-glycogen synthase kinase (pGSK-3 $\beta ; 1: 100$, ab107166, Abcam, Cambridge, MA, USA), phosphorylated-extracellular signal-regulated kinase (pERK)1/2 (Thr202/Tyr204; 1:1,000, 9101, Cell Signaling Technology), B-cell lymphoma 2 (Bcl-2; 1:100, sc-7382, Santa Cruz Biotechnology, Dallas, TX, USA), Bcl-2 associated X (Bax; 1:100, ab5714, Abcam), nestin (1:200, ab6142, Abcam), neuronal nuclei (NeuN; 1:100, MAB377, Millipore, Bedford, MA, USA), doublecortin (DCX; 1:100, ab28941, Abcam), and SRY-box transcription factor 2 (SOX2; 1:50, ab79351, Abcam; 1:50, ab97959, Abcam). The detailed methods are described in the Supplementary methods.

\section{Western blotting}

The peri-infarct region in the control (sham and saline) group and the corresponding area in the GV1001-treated group, which were confirmed based on MRI, were used in Western blot analysis for phospho-insulin receptor substrate-1 (pIRS-1) (Ser636/639; 1:1,000, 2388, Cell Signaling Technology), phospho-phosphoinositide 3-kinase (pPI3K) p85 (Tyr458)/p55(Tyr199) (p85a PI3K; 1:1,000, 4228, Cell Signaling Technology), pAkt (Ser473), Akt (1:2,000, 9272, Cell Signaling Technology), pGSK-3 $\beta$ (1:1,000, 9336, Cell Signaling Technology), GSK-3 $\beta$ (1:2,000, sc-9166, Santa Cruz Biotechnology), pERK1/2 (Thr202/ Tyr204), Bcl-2, Bax (1:1,000, 2772, Cell Signaling Technology), uridine diphosphate glucuronosyltransferase 1A1 (UGT1A1, 1:500, ab237810, Abcam), FK506 binding protein 5 (FKBP5, 0.4 $\mu \mathrm{g} / \mathrm{mL}$, NBP1-84676, Novus Biologicals, Centennial, CO, USA), sphingosine kinase type 1 (SPHK1, 1:1,000, ab71700, Abcam), and beta-tubulin ( $\beta$-tubulin, 1:2,000, 2146, Cell Signaling Technology). The detailed method for Western blotting is described in the Supplementary methods.

\section{In vitro methods}

\section{Culture and treatment of NSCs and primary cortical neu- rons}

NSCs were isolated from rodent embryonic brains, cultured, and then expanded. NSCs were cultured as described previously. ${ }^{12,13}$ Primary cultured cortical neurons were obtained from the cerebral cortex of fetal Sprague-Dawley rats (Orient Bio, Seongnam, Korea) on embryonic day 16. The methods for culturing NSCs and primary cortical neurons are briefly described 
in the Supplementary methods.

OGD was achieved in a Forma anaerobic chamber (Thermo Fisher Scientific, Waltham, MA, USA). A gas mixture containing $5 \% \mathrm{CO}_{2}, 0.2 \% \mathrm{O}_{2}$, and $94.8 \% \mathrm{~N}_{2}$ was flushed through the chamber for $1,2,4,6,8,10,12,14,16,22$, and 24 hours. This procedure maintained a non-fluctuating hypoxic environment of $<1 \% \mathrm{O}_{2}$. Reoxygenation was performed after 1 and 8 hours of incubation in a hypoxic chamber, and the cells were incubated again in a normoxic incubator $\left(95 \%\right.$ air $\left./ 5 \% \mathrm{CO}_{2}\right)$ for 8 hours at $37^{\circ} \mathrm{C}$. Untreated cells served as controls.

To evaluate the effect of GV1001 on NSCs, the cells were treated with several concentrations of GV1001 alone. Finally, to measure the protective effect of GV1001 against OGD, NSCs were treated with several concentrations of GV1001 under OGD for 1 and 8 hours, as described above.

\section{Molecular studies}

The diverse molecular analyses included (1) trypan blue staining (TBS) and lactate dehydrogenase (LDH) release assay to measure cell viability and cytotoxicity; (2) bromodeoxyuridine (BrdU) cell proliferation assay; (3) colony-forming unit (CFU) assay to determine cell growth; (4) annexin $\mathrm{V}$ and propidium iodide staining to detect cell death; (5) evaluation of apoptosis; (6) determination of cell migration; (7) determination of free radical production; (8) malondialdehyde (MDA) assay of lipid peroxidation due to oxidative stress; (9) determination of $\mathrm{Ca}^{2+}$ level; (10) determination of oxidative mitochondrial DNA damage; (11) adenosine triphosphate (ATP) assay; (12) mitochondrial membrane potential assay; (13) Western blot analysis; (14) proteomics analysis; and (15) antibody microarrays. The methods are detailed in the Supplementary methods.

\section{Statistical analyses}

All data are presented as the mean \pm standard deviation values from five or more independent experiments. Statistical analyses of three or more datasets were performed using one-way analysis of variance, followed by Tukey's post hoc comparisons. Statistical significance was set at $P<0.05$. All statistical analyses were performed using IBM SPSS Statistics for Windows version 21.0 (IBM Co., Armonk, NY, USA).

\section{Results}

\section{In vivo results}

\section{Reduction of infarct volume by GV1001}

Administration of GV1001 2 hours after MCAO attenuated the infarct volume at 48 hours. The effective doses of GV1001 were
30 and $60 \mu \mathrm{M} / \mathrm{kg}$, which were used in subsequent experiments (Figure 1B). The initial infarct volumes on DWI were not significantly different between the GV1001-treated and saline-treated groups $(P=0.278)$. The infarct volume on FLAIR MRI 48 hours after MCAO compared to lesion volume on DWI MRI just before injection showed a significantly smaller ratio in the GV1001-treated group than in the saline-treated group (Figure 1C).

Efficacy of GV1001 based on behavioral evaluation and expression of TUNEL-positive cells

The GV1001-treated groups showed consistently better neurological outcomes than the saline group. The treatment effects over time were significant in a dose-dependent manner for the rotarod, modified adhesive-removal, and beam balance tests (Figure 1D). After sacrificing the rats, brain tissues were sectioned and analyzed by TUNEL assay to assess apoptotic cell death. The number of TUNEL-positive cells in the ischemic periinfarct region was compared among the sham, saline, and GV1001-treated groups (30 and $60 \mu \mathrm{M} / \mathrm{kg}$ GV1001). The number of TUNEL-positive cells was reduced in the GV1001-treated group compared with the control group in a dose-dependent manner (Figure 1E).

\section{Effect of GV1001 on intracellular signals}

To investigate the mechanisms underlying GV1001-induced neuroprotection against ischemic stroke, the immunoreactivity of pIRS-1, pAkt (Ser473), pGSK-3 $\beta$ (Ser9), pERK1/2, Bcl-2, Bax, glial fibrillary acidic protein (GFAP, an astrocyte marker), nestin (a neuroectodermal stem cell marker), NeuN (a neuronal nuclear antigen), DCX (a neuronal differentiation marker), and SOX2 (a multipotent NSC marker) were measured. Immunoreactivities of survival-related proteins (pIRS-1, pAkt, pGSK-3 $\beta$, pERK1/2, and $\mathrm{Bcl}-2$ ) were significantly increased in the periinfarct region after treatment with GV1001. The expression of Bax, which is involved in cell death, was decreased by treatment with GV1001 (Figure 2A).

Immunohistochemistry confirmed the increases in pAkt (Ser473), pGSK-3ß (Ser9), pERK, and Bcl-2 levels and decreases in Bax levels along with increment in the number of NeuN- or SOX2-positive cells after treatment with GV1001 (Figure 2B and Supplementary Figure 1). Additionally, increased levels of nestin, NeuN, SOX2, and DCX were noted in the peri-infarct subventricular zone of the GV1001-treated group, but GFAP expression was decreased by GV1001 treatment (Figure 2C). The levels of markers for neurotoxic phenotype reactive astrocytes (UGT1A1 and FKBP5) were increased and those of neuroprotective phenotype reactive astrocytes (SPHK1) were de- 

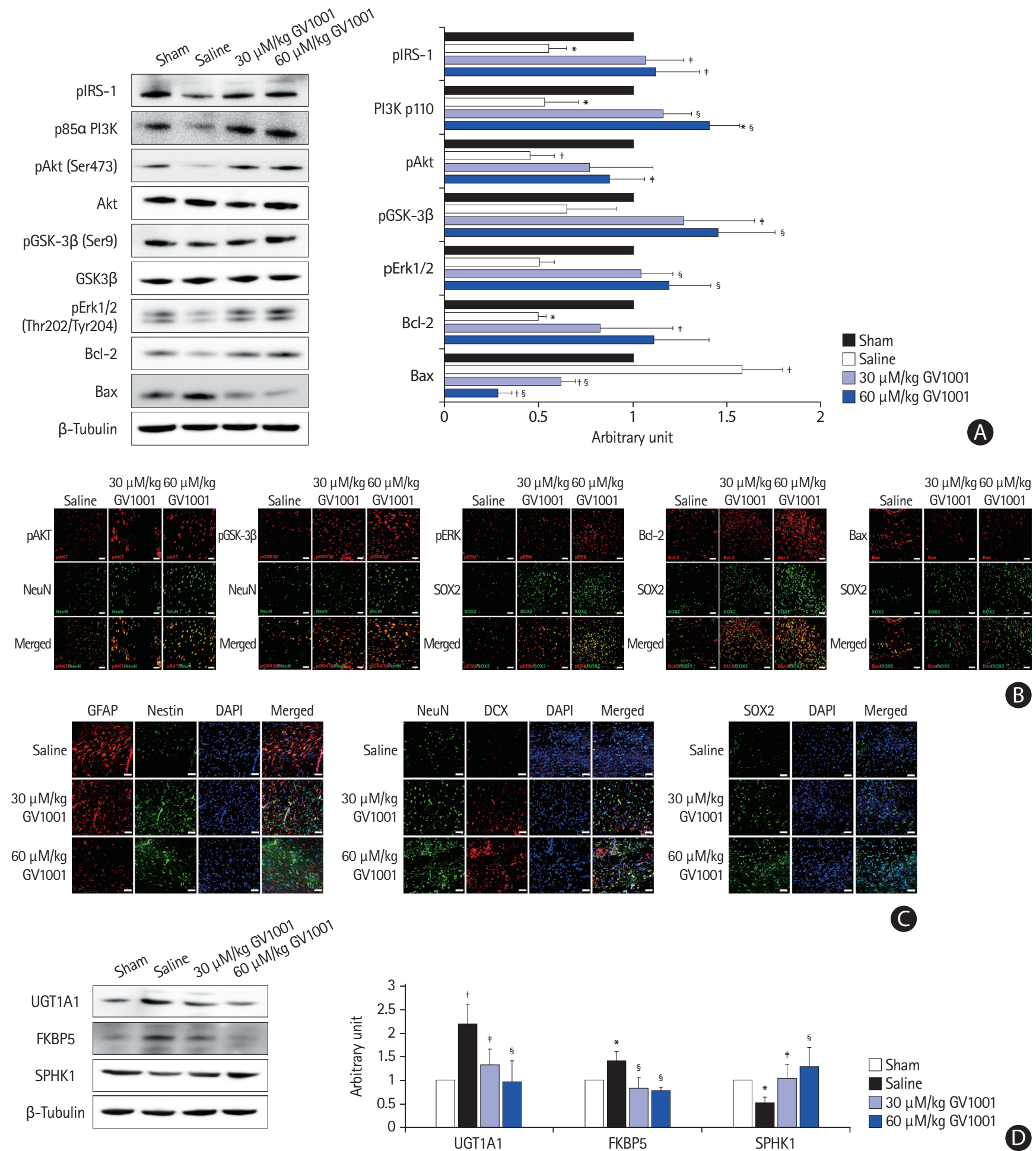

Figure 2. The effect of GV1001 on intracellular signaling proteins and cells. (A) Decreased levels of phosphorylated Akt (pAkt; Ser473), phospho-glycogen synthase kinase (pGSK-3ß; Ser9), phosphorylated-extracellular signal-regulated kinase (pERK), B-cell lymphoma 2 (Bcl-2), and increased levels of Bcl-2 associated X (Bax) were noted in the peri-infarct regions of GV1001-treated rats compared to those of control rats. Administration of GV1001 (30 and $60 \mu \mathrm{M} / \mathrm{kg})$ alleviated the changes. (B) Immunohistochemistry (IHC) staining showing increased pAkt (Ser473), pGSK-3 $\beta$ (Ser9), pERK, Bcl-2 levels and decreased Bax levels along with increased numbers of neuronal nuclei (NeuN)- or SRY-box transcription factor 2 (SOX2)-positive cells in GV1001-treated groups, scale bar: $50 \mu \mathrm{m}$. (C) IHC also revealed increased levels of nestin (a neuroectodermal stem cell marker), NeuN (a neuronal nuclear antigen), doublecortin (DCX; a neuronal differentiation marker), and SOX2 (a multipotent neural stem cell marker) in the peri-infarct region of GV1001-treated rats. Glial fibrillary acidic protein (GFAP; an astrocyte marker) expression was decreased in GV1001-treated rats, scale bar: $50 \mu \mathrm{m}$. (D) Levels of neurotoxic phenotype reactive astrocytes (uridine diphosphate glucuronosyltransferase 1A1 [UGT1A1] and FK506 binding protein 5 [FKBP5]) were increased and those of neuroprotective phenotype reactive astrocytes (sphingosine kinase type 1 [SPHK1]) were decreased in the peri-infarct area, but GV1001 significantly restored the expression of these markers. pIRS-1, phospho-insulin receptor

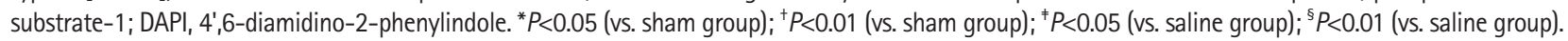



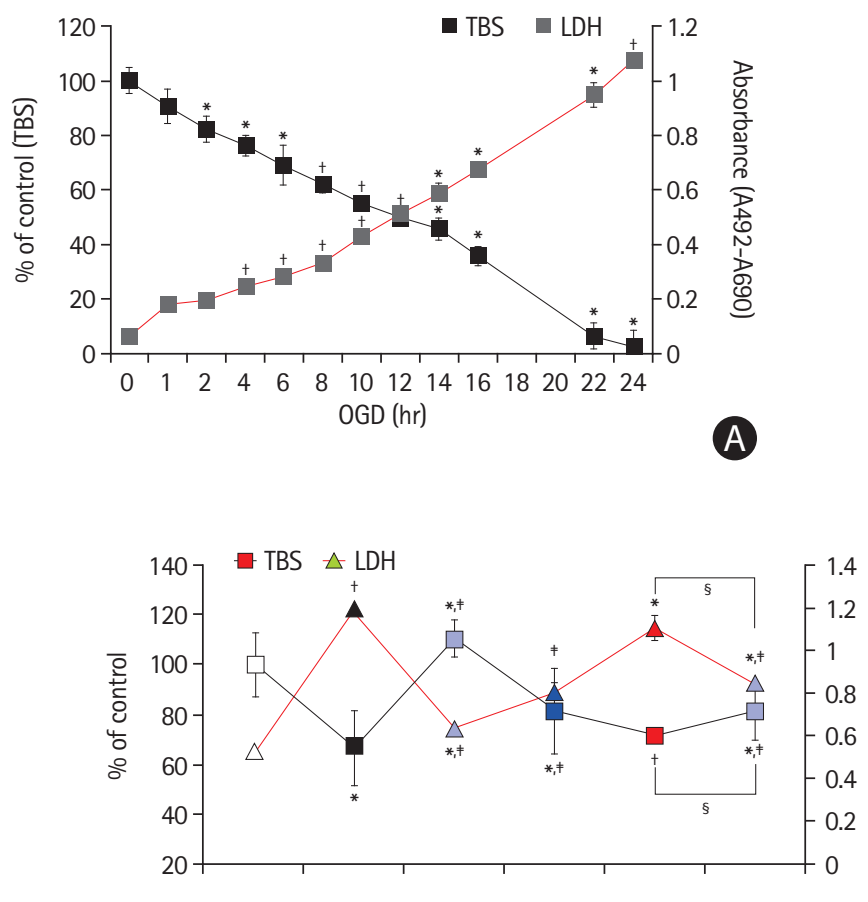

A

GV1001 $(\mu \mathrm{M})$

$8 \mathrm{hr}$ reoxygenation

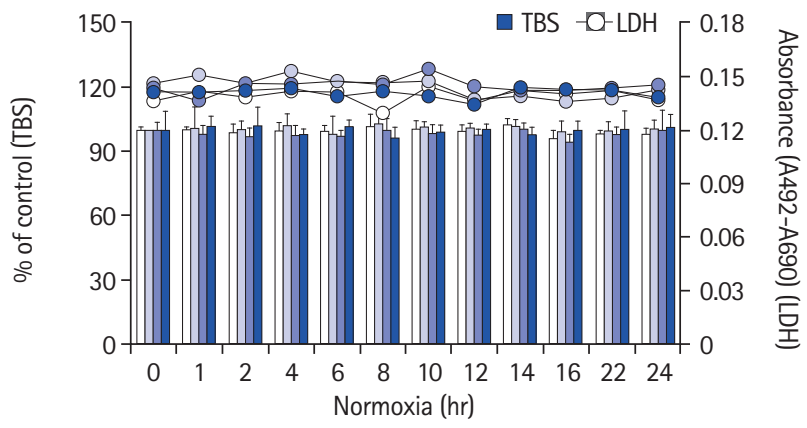

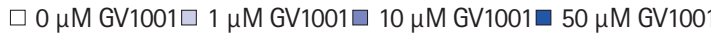

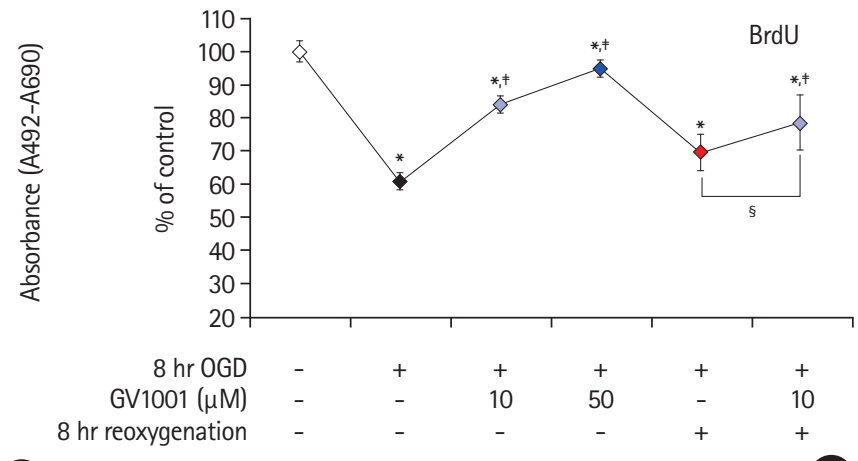

C

$8 \mathrm{hr} \mathrm{OGD}$

$0 \mu \mathrm{M}$ GV1001 $0 \mu \mathrm{M}$ GV1001 $10 \mu \mathrm{M}$ GV1001 $50 \mu \mathrm{M}$ GV1001

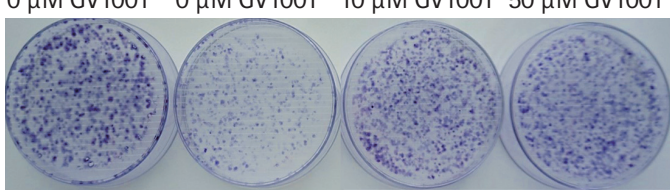

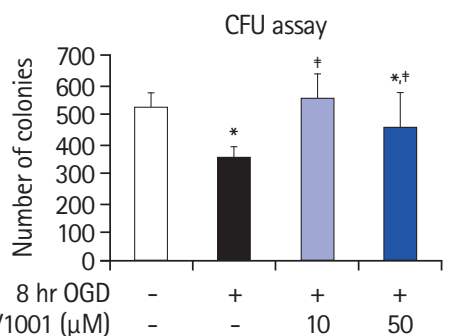

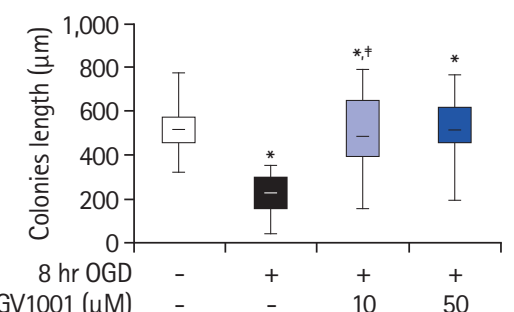

E

$8 \mathrm{hr}$ OGD

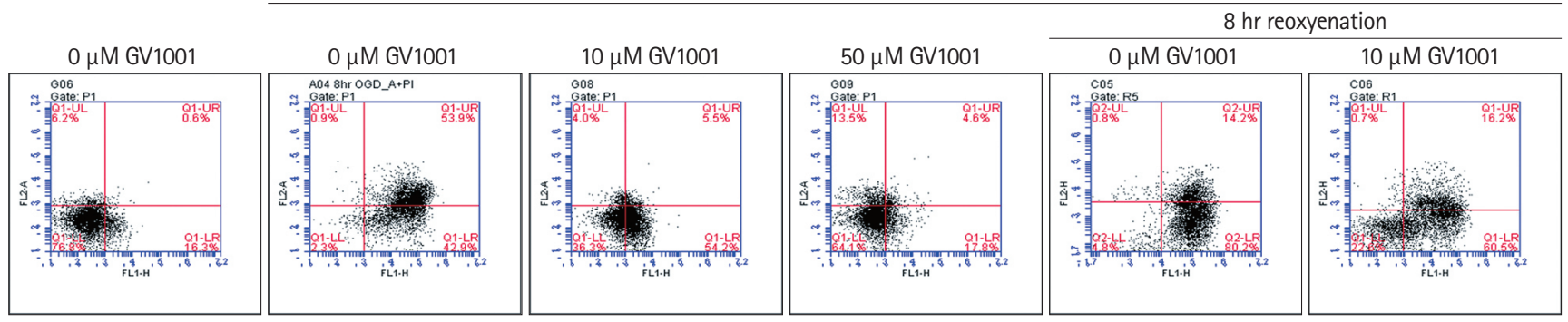

Figure 3. Effect of GV1001 on viability, cytotoxicity, and proliferation of neural stem cells (NSCs) after treatment with oxygen-glucose deprivation (OGD) alone or 0GD/reperfusion (OGD/R). (A) OGD induced cell death and cytotoxicity in a time-dependent manner. (B) GV1001 at concentrations of up to $50 \mu \mathrm{M}$ did not affect the viability and cytotoxicity of NSCs. (C) Treatment with GV1001 increased cell viability and decreased cytotoxicity of NSCs injured by OGD/R. (D, E) To evaluate the effects of prolonged OGD/R and/or varying concentrations of GV1001 on cell proliferation, NSCs were exposed to OGD/R for 8 hours with different concentrations of GV1001 and were incubated in bromodeoxyuridine (BrdU) labeling medium. CFU assays showed that proliferation was inhibited in OGD/R-injured NSCs but was markedly restored with GV1001 treatment. (F) Various viability tests also showed that GV1001 increased the viability of NSCs after injury by OGD/R. (G) 4',6-Diamidino-2-phenylindole (DAPI) and transferasemediated deoxyuridine triphosphate nick end labeling (TUNEL) staining revealed the anti-apoptotic effect of GV1001 on OGD/R-injured NSCs, scale bar: $25 \mu$ m. (H) Cell migration assay showed that treatment with GV1001 restored cell migration activity of NSCs that had been reduced by 0GD/R. TBS, trypan blue staining; LDH, lactate dehydrogenase; CFU, colony-forming unit. ${ }^{P} P<0.05$ (vs. control group); ${ }^{+} P<0.01$ (vs. control group); ${ }^{\dagger} P<0.05$ (vs. NSCs only treated with $0 \mathrm{GD}$ ); ${ }^{\circledR} P<0.05$ (vs. NSCs treated with $0 \mathrm{GD} / \mathrm{R}$ ). 

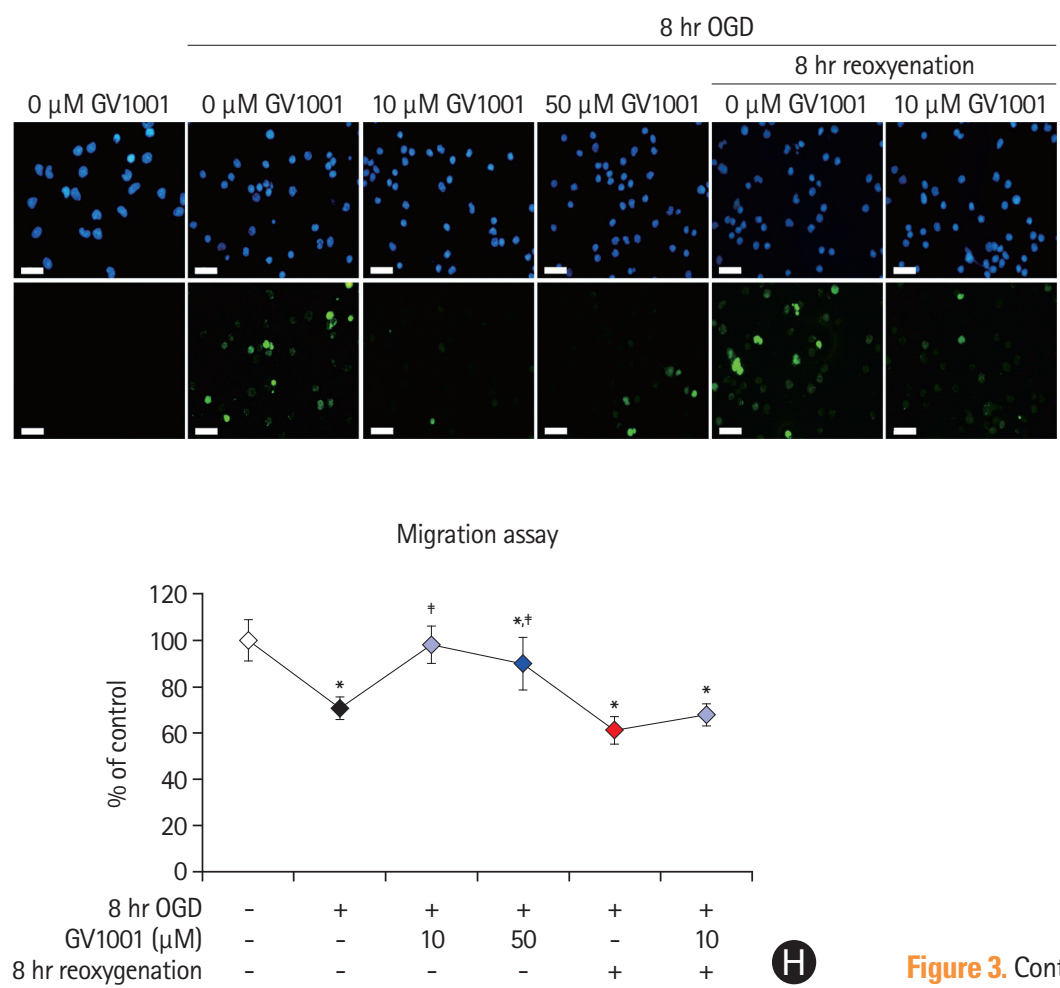

creased in the peri-infarct area of the MCAO rat model, and GV1001 significantly restored the expression of these markers (Figure 2D).

\section{In vitro results}

Effect of OGD/R and GV1001 on NSC viability, proliferation, and mobilization

Cell viability was measured using TBS and LDH assays. Exposure of primary cultured NSCs to OGD for 1 to 24 hours significantly reduced NSC viability in a time-dependent manner (Figure $3 \mathrm{~A}$ ). As cell viability was $60 \%$ to $70 \%$ after 8 hours of $\mathrm{OGD}$ exposure, this condition was considered as optimal duration (Figure 3A). GV1001 alone did not affect the viability of NSCs (Figure 3B).

NSCs were exposed to OGD for 8 hours and treated simultaneously with different concentrations of GV1001. Compared with treatment with $\mathrm{OGD} / \mathrm{R}$ alone, treatment with $\mathrm{OGD} / \mathrm{R}$ and GV1001 at concentrations of 10 and $50 \mu \mathrm{M}$ increased NSC viability, as measured by the TBS and LDH assays (Figure $3 \mathrm{C}$ and F), and proliferation, as measured by the BrdU and CFU assays (Figure 3D and E).

The effect of GV1001 on the apoptosis of NSCs induced by OGD/R was determined by 4',6-diamidino-2-phenylindole (DAPI) and TUNEL staining. These results revealed that the percentage of apoptotic cells was increased by OGD/R and

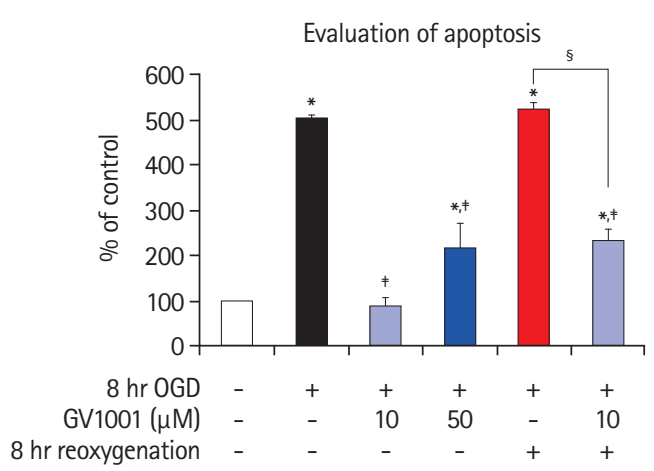

G

Figure 3. Continued.

was significantly decreased by co-treatment with GV1001 (up to $50 \mu \mathrm{M}$ ) (Figure 3G). Migratory capacity, which is an important characteristic of stem cells, was evaluated using a migration assay kit. $0 G D / R$ reduced the migratory capacity of NSCs, but co-treatment with GV1001 (10 and $50 \mu \mathrm{M}$ ) effectively restored this capacity (Figure $3 \mathrm{H}$ ).

Effect of GV1001 on free radical production and oxidative mitochondrial DNA damaged by OGD/R

We evaluated the free radical scavenging effect of GV1001 after OGD. GV1001 alone did not trigger reactive oxygen species (ROS) production under cell-free conditions (Figure 4A). ROS levels were dramatically elevated by reoxygenation after OGD in NSCs, and GV1001 significantly inhibited ROS production (Figure 4B). The levels of MDA, a lipid peroxidation marker, were measured to confirm the antioxidant effect. The MDA levels in NSCs were increased by OGD/R. However, GV1001 effectively decreased this level in a concentration-dependent manner (Figure 4C). Intracellular $\mathrm{Ca}^{2+}$ levels decreased, and oxidative mitochondrial DNA damage increased with OGD/R. These effects were significantly reversed by the administration of GV1001 (Figure 4D and E).

\section{Effect of GV1001 on mitochondria of NSCs injured by $O G D / R$}

We measured ATP levels and mitochondrial membrane poten- 

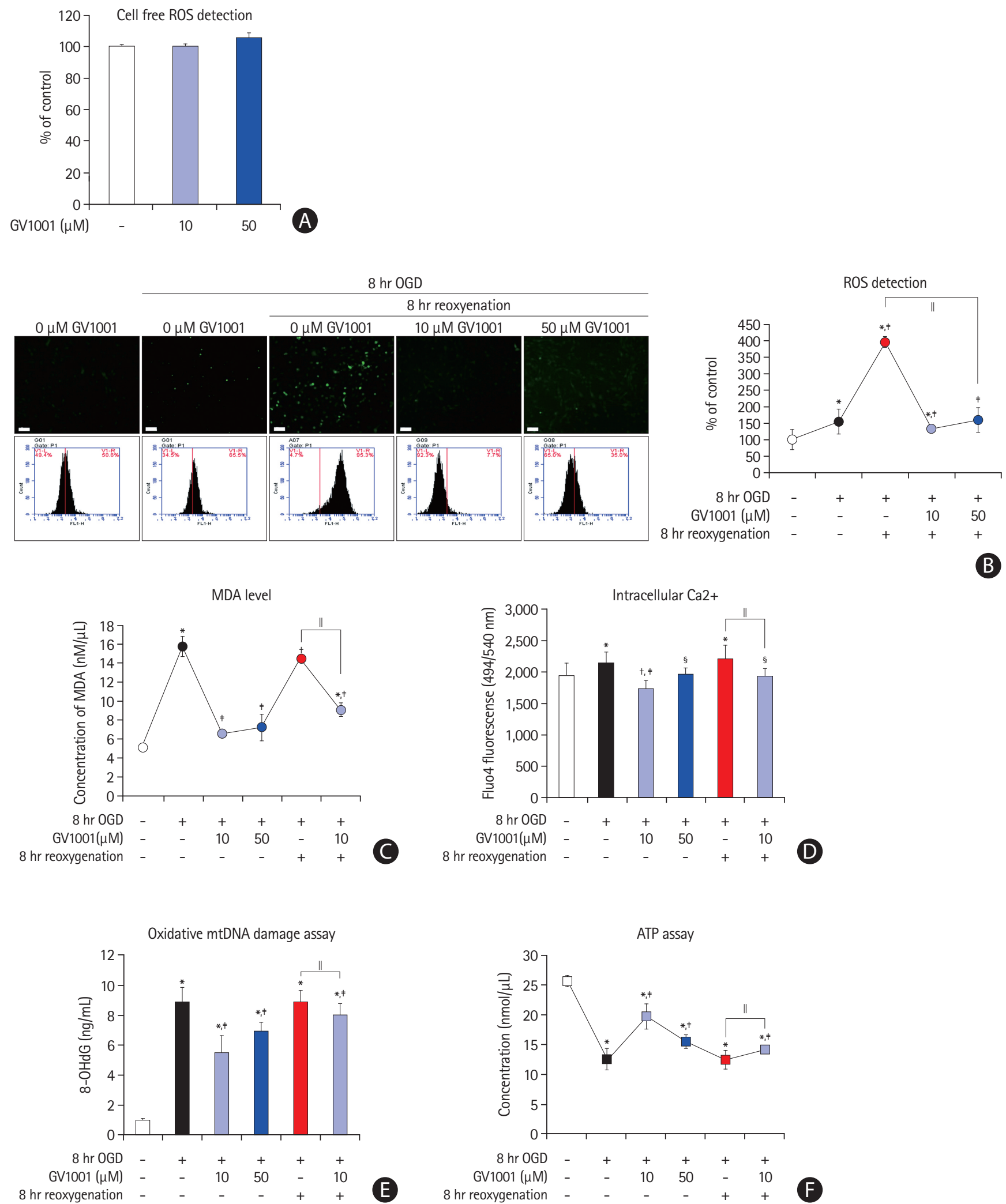

Figure 4. (A) Antioxidant effect of GV1001. GV1001 alone did not trigger reactive oxygen species (ROS) production. (B) Intracellular ROS produced after oxygenglucose deprivation/reperfusion (OGD/R) was decreased after treatment with GV1001, scale bar: $25 \mu \mathrm{m}$. (C, D, E) The levels of malondialdehyde (MDA) and intracellular $\mathrm{Ca}^{2+}$ and oxidative mitochondrial DNA damage were increased by OGD/R and were significantly decreased upon GV1001 treatment. (F) Adenosine triphosphate (ATP) assay for the function of mitochondria. ATP levels were decreased by OGD/R. GV1001 restored ATP levels. (G) The mitochondrial membrane potential was significantly decreased with $0 G D / R$, but GV1001 markedly restored this potential. mtDNA, mitochondrial deoxyribonucleic acid. ${ }^{*} P<0.05$ (vs. control group); ${ }^{+} P<0.01$ (vs. control group); ${ }^{\dagger} P<0.05$ (vs. NSCs only treated with 0 GD); ${ }^{\circledR} P<0.01$ (vs. NSCs only treated with $0 G D$ ); $" ~ P<0.05$ (vs. NSCs treated with 0 GD/R). 

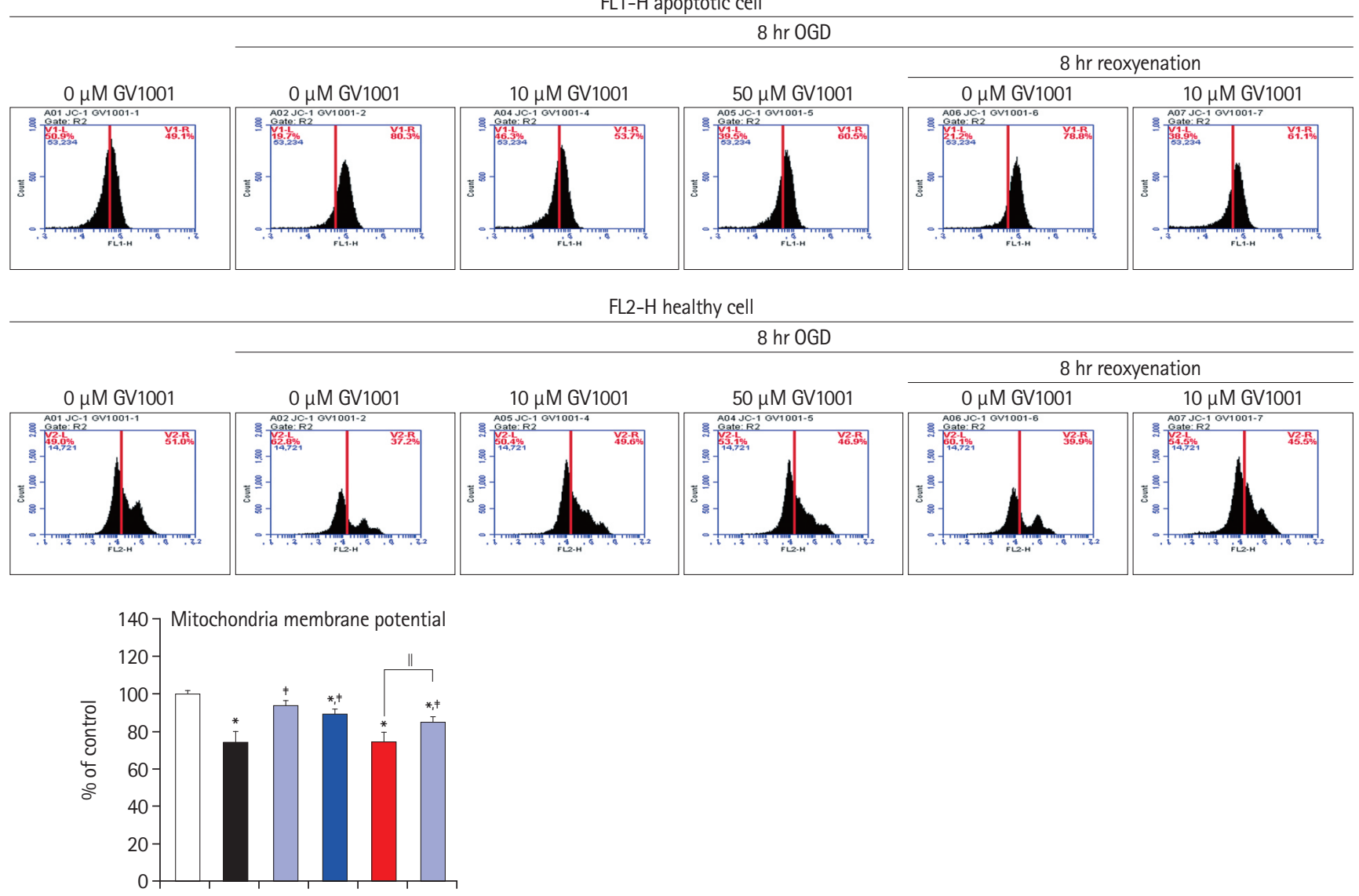

$8 \mathrm{hrOGD}-++\quad+\quad+\quad+$ GV1001 $(\mu \mathrm{M}) \quad-\quad-\quad 1050 \quad-\quad 10$

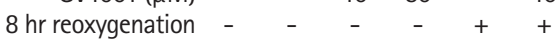

Figure 4. Continued.

tials to evaluate the effect of GV1001 on the mitochondria of NSCs injured by OGD/R. ATP levels were reduced in NSCs after $O G D / R$, but this reduction was significantly alleviated by GV1001 treatment (Figure 4F). The mitochondrial membrane potential in NSCs was decreased by OGD/R but was significantly restored by GV1001 (Figure 4G).

\section{Effect of OGD/R and GV1001 on intracellular protein levels}

We performed Western blot and immunocytochemistry analyses to directly measure changes in the immunoreactivities of proteins affected by treatment with OGD/R and GV1001 (Figure 5A-C). Ki67 level, which is associated with proliferative activity, was decreased by OGD/R but was increased by GV1001 (Figure $5 A)$. The levels of survival-related proteins, such as p85a, PI3K, pAkt (Ser 473), pGSK-3 $\beta$ (Ser9), and cytoplasmic high mobility group box protein 1 (HMGB-1), were also decreased following 0GD/R injury but were recovered with GV1001 treatment (Figure $5 \mathrm{~A}$ ). The levels of death-related proteins, such as Bax, cytosolic cytochrome c, cleaved caspase-3, and cleaved caspase-9, were increased with $0 G D / R$ but were significantly decreased with GV1001 treatment (Figure 5B).

We also measured alterations in the Wnt signaling pathway to examine the non-canonical telomerase activity. $0 G D / R$ decreased active (non-phospho) $\beta$-catenin expression in the cytoplasm, whereas GV1001 significantly increased active $\beta$-catenin expression. OGD/R significantly decreased the expression of cyclin D1 and c-Myc, but GV1001 effectively restored their expression, although the expression pattern of c-Myc was restored slightly at 1 hour after treatment (Figure 5C). Additionally, proteomic analyses were performed to determine the effect of OGD/R and GV1001 on the production of intracellular proteins in NSCs. The production of numerous proteins was altered by $\mathrm{OGD} / \mathrm{R}$, and treatment with GV1001 restored the altered protein production (Figure 5D). An antibody microarray was performed to assess the phosphorylation patterns of proteins. The levels of the phosphorylated forms of various survival-related proteins were decreased by 8 hours of OGD/R and were restored by GV1001 treatment. The level of tryptophan hydroxylase, which catalyzes serotonin synthesis, was increased by $O G D / R$ and decreased by GV1001 treatment (Figure 5E). 

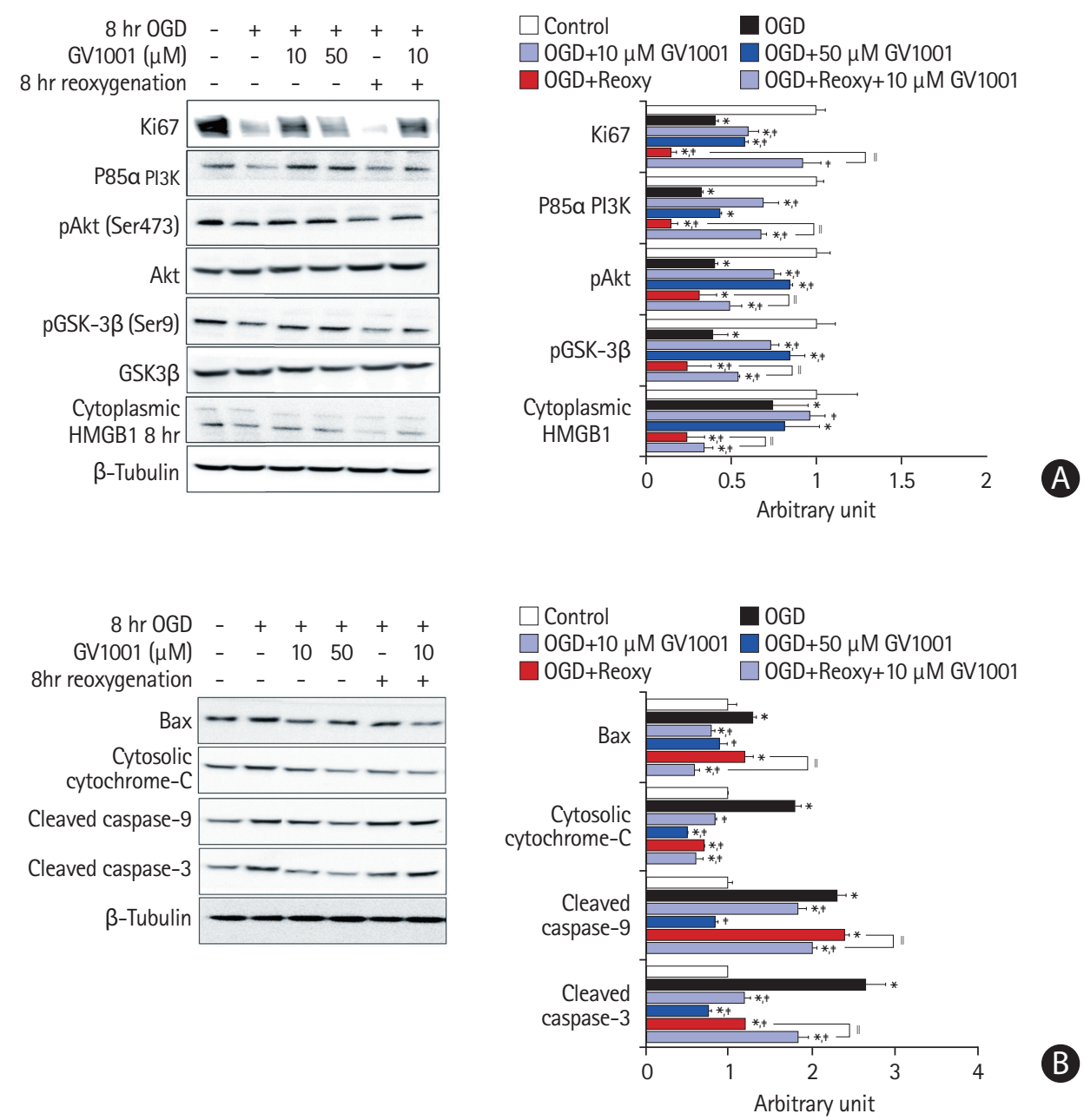

Figure 5. (A) Effect of GV1001 on intracellular proteins in oxygen-glucose deprivation/reoxygenation (OGD/R)-injured neural stem cells (NSCs). Western blots showed that the levels of proteins associated with the proliferation and survival of NSCs decreased with OGD/R. The protein levels were increased upon treatment with GV1001. (B) Levels of proteins associated with cell death increased with OGD/R. These levels were decreased upon GV1001 treatment. (C) Expressions of active $\beta$-catenin, cyclin D1, and C-myc were evaluated to confirm the non-canonical telomerase activity of GV1001. These levels were decreased after OGD/R and were restored by GV1001 treatment. The data are expressed as percentages of the value in the control group and represented as the mean \pm standard deviation values from five independent experiments. (D) Proteomic and (E) antibody microarray data revealed that GV1001 affected the levels of numerous intracellular proteins in OGD/R-injured NSCs. PI3K, phosphoinositide 3-kinase; pAkt, phosphorylated Akt; pGSK-3ß, phospho-glycogen synthase kinase; HMGB1, high mobility group box protein $1 ; \mathrm{Bax}, \mathrm{Bcl}-2$ associated $\mathrm{X} ; \mathrm{HSP}$, heat shock protein; ATP, adenosine triphosphate; PP1, protein phosphatase $1 ;$ NADH, nicotinamide adenine dinucleotide hydride; COP9, constitutive photomorphogenesis 9; $C A A$, coenzyme $A ; G R P 1$, general receptor of phosphoinositides 1; ARNO3; ARF nucleotide-binding site opener 3; ERK, extracellular signal-regulated kinase; b-NOS, brain nitric oxide synthase; PKB, protein kinase $B ;$ PKC, protein kinase $C$. ${ }^{*} P<0.05$ (vs. control group); ${ }^{+} P<0.01$ (vs. control group); ${ }^{*} P<0.05$ (vs. NSCs only treated with 0 GD); ${ }^{5} P<0.01$ (vs. NSCs only treated with 0 GD); ${ }^{~} P<0.05$ (vs. NSCs treated with $0 G D / R)$.

Critical roles of PI3K pathway in GV1001-induced rejuvenation of stem cell characteristics of OGD/R-injured NSCS OGD/R significantly deteriorated the stem cell characteristics, including survival, proliferation, and migration of NSCs. However, GV1001 effectively counteracted these effects and restored the PI3K pathway. To investigate the role of the PI3K pathway in the rejuvenation of NSCs by GV1001, NSCs were pretreated with the PI3K inhibitor LY294002 before OGD/R and GV1001 treatments. Pretreatment with the PI3K inhibitor blocked the effect of GV1001 on OGD-injured NSCs, and cell viability was significantly decreased compared to that of NSCs not pretreated with the PI3K inhibitor (Figure 6). However, in NSCs injured with OGD/R (8 hours OGD with 8 hours reoxygenation), the PI3K inhibitor attenuated the protective effect of GV1001, but the effect was not statistically significant.

\section{Effect of GV1001 on cortical neurons damaged by OGD/R}

To confirm the effect of GV1001 on neurons injured by OGD/R, viability, toxicity, and ROS production were assessed (Figure 7). GV1001 restored the viability and reduced the toxicity of cortical neurons, which were injured by OGD/R (Figure 7A and B). Furthermore, GV1001 significantly inhibited the production of 

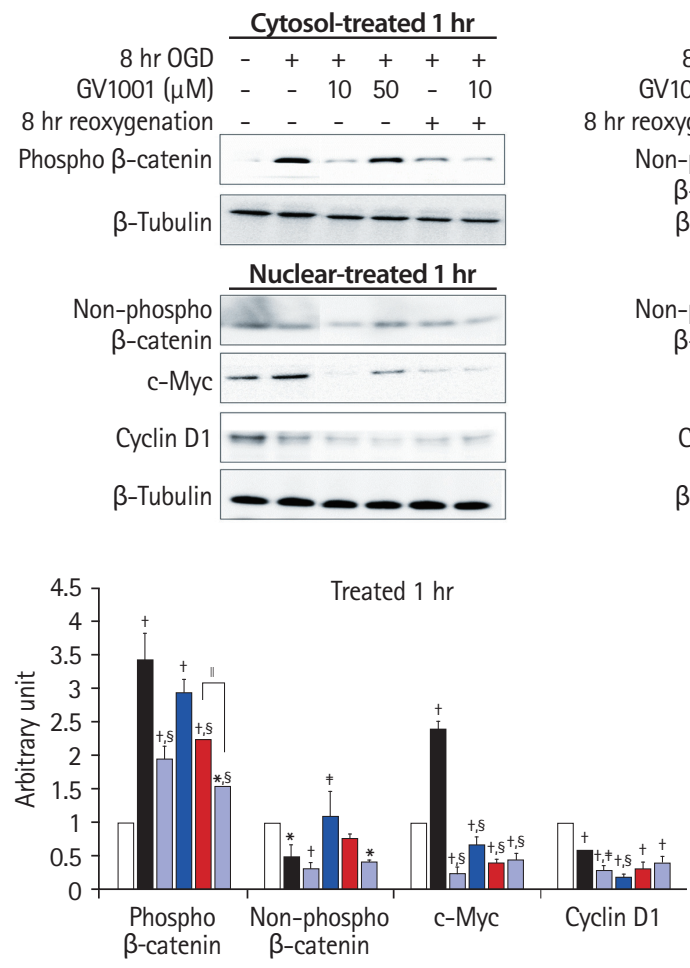
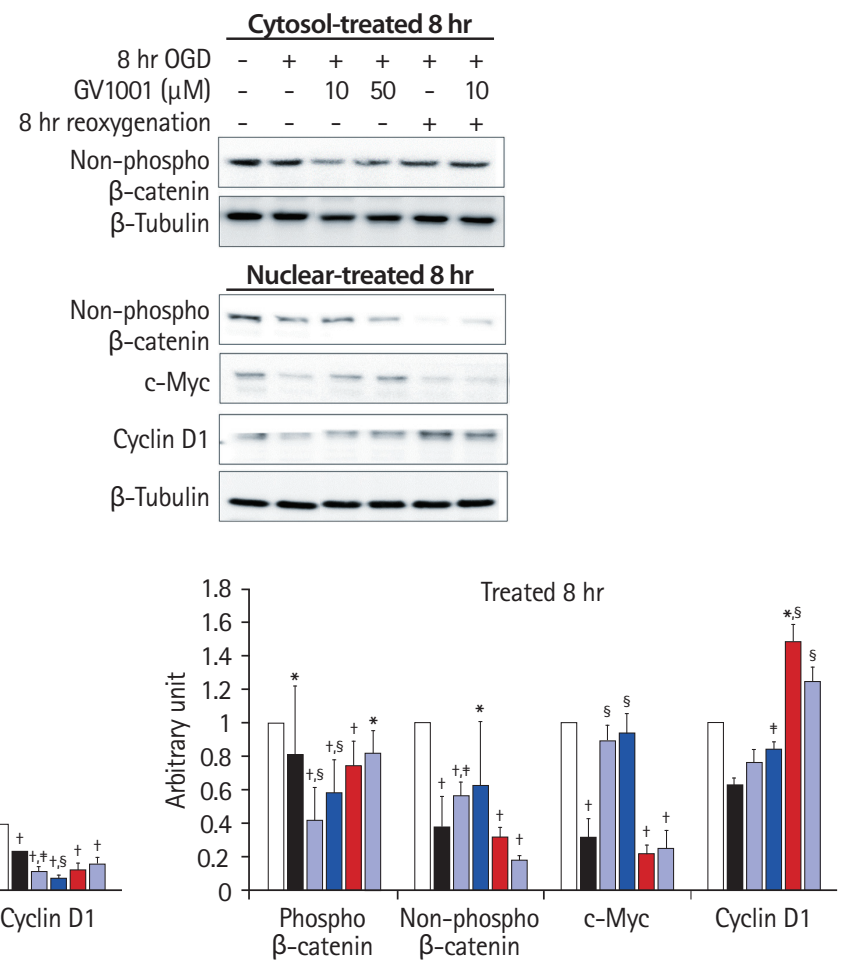

$\square$ Control

$\square$ 0GD+10 $\mu \mathrm{M}$ GV1001

OGD+Reoxy

OGD

$\square$ 0GD+50 $\mu \mathrm{M}$ GV1001

$\square$ OGD+Reoxy+10 $\mu \mathrm{M}$ GV1001

$8 \mathrm{hr} \mathrm{OGD}$
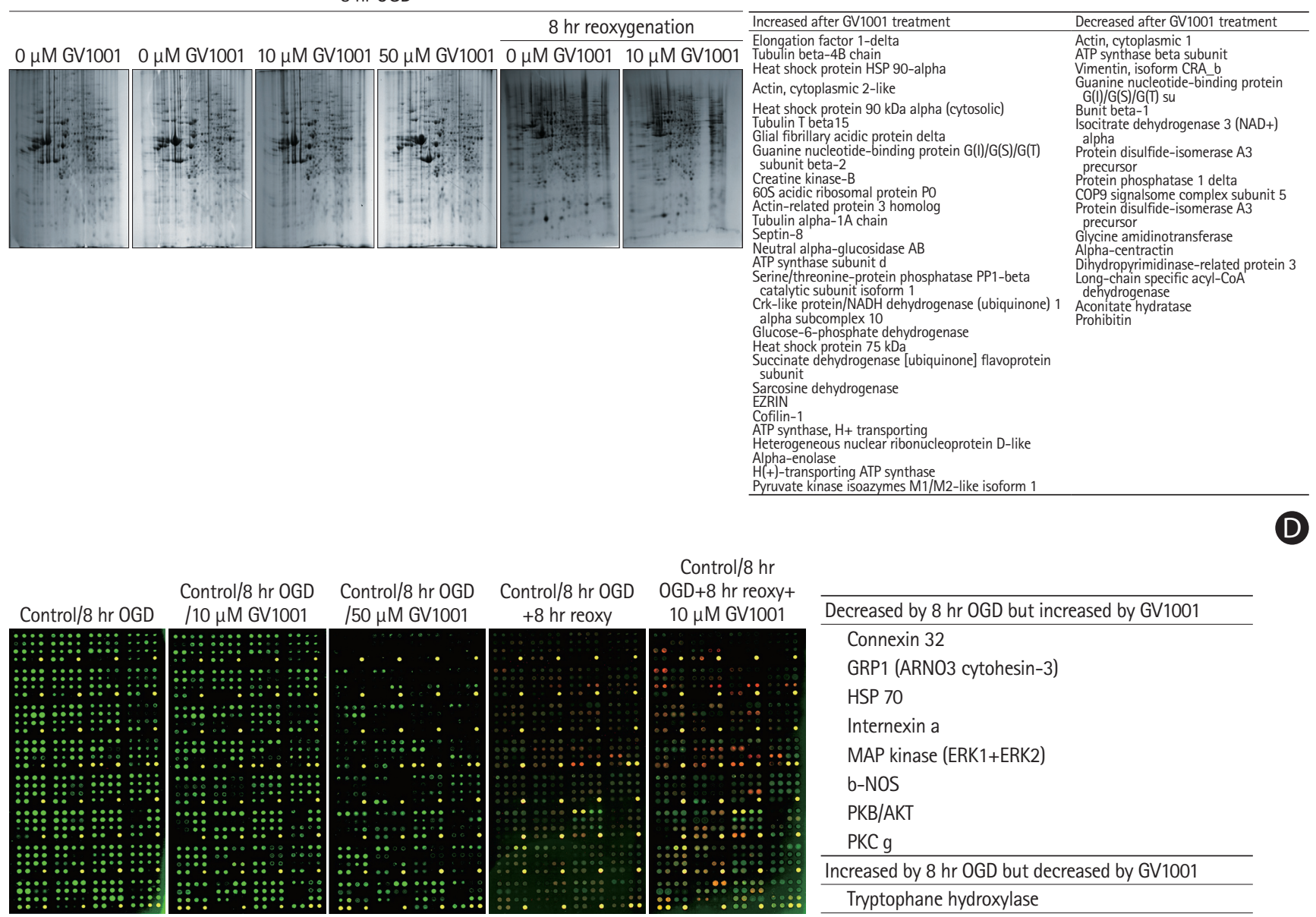

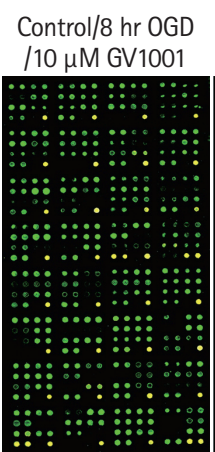

Control/8 hr OGD

Control/8 hr OGD

Control/8 hr

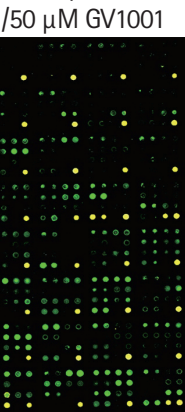

$+8 \mathrm{hr}$ reoxy

OGD+8 hr reoxy+
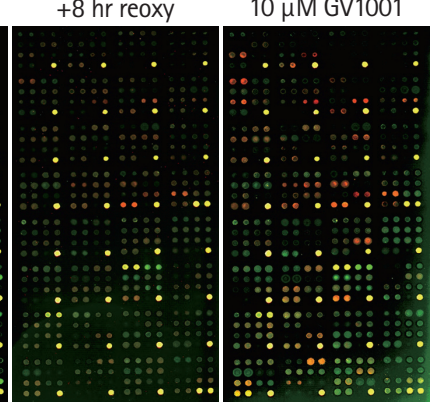

\begin{tabular}{l}
\hline Decreased by 8 hr OGD but increased by GV1001 \\
\hline Connexin 32 \\
GRP1 (ARN03 cytohesin-3) \\
HSP 70 \\
Internexin a \\
MAP kinase (ERK1+ERK2) \\
b-NOS \\
PKB/AKT \\
PKC $g$ \\
\hline Increased by 8 hr OGD but decreased by GV1001 \\
\hline Tryptophane hydroxylase
\end{tabular}

Figure 5. Continued. 
Cell viability

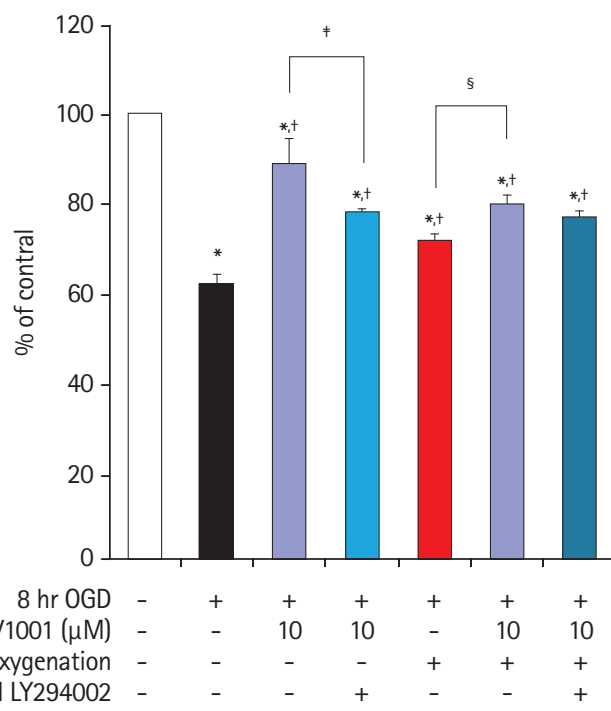

Figure 6. Oxygen-glucose deprivation/reoxygenation (OGD/R)-injured neural stem cells (NSCs) were treated with the phosphoinositide 3-kinase (PI3K) inhibitor LY294002 to assess the role of the PI3K/Akt pathway in the protective mechanism of GV1001. The NSCs were divided into seven groups: control (group 1); OGD (group 2); OGD+10 $\mu \mathrm{M}$ GV1001 (group 3);

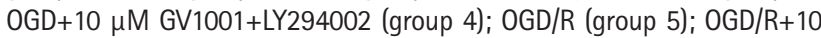
$\mu \mathrm{M}$ GV1001 (group 6); and 0GD/R+10 $\mu \mathrm{M}$ GV1001+LY294002 (group 7). LY294002 significantly blocked the effects of GV1001 on OGD-injured NSCs. ${ }^{*} P<0.05$ (vs. group 1); ${ }^{+} P<0.05$ (vs. group 2); ${ }^{\circledR} P<0.05$ (group 3 vs. group 4); ${ }^{\circledR} P<0.05$ (group 5 vs. group 6).

ROS, which was elevated by reoxygenation after OGD, in these neurons (Figure 7C). The levels of survival-related proteins were decreased and those of death-associated proteins were increased by OGD/R. However, treatment with GV1001 reversed these changes (Figure 7D).

\section{Discussion}

In the present study, we demonstrated that GV1001 reduced the infarct volume and restored the neurologic function in an ischemic stroke rat model. GV1001 stimulated neuroprotective pathways, as demonstrated by the increased levels of $p A k t$, pGSK- $3 \beta$, pERK $1 / 2$, and $B c l-2$, and decreased levels of Bax along with an increase in the number of NeuN-positive neurons and SOX2-positive NSCs. The expression of death-related proteins, including cleaved caspase- 3 , cleaved caspase- 9 , and Bax, was suppressed by GV1001. The in vitro OGD/R model demonstrated that GV1001 protected NSCs from OGD/R injury by improving viability, restoring proliferation, increasing mobilization, stabilizing mitochondria, and exerting antioxidant effects. In addition, GV1001 improved viability, increased migration activity, and decreased ROS in OGD/R-injured cortical neurons.
GV1001 is a vaccine peptide derived from $\mathrm{hTERT}{ }_{1}{ }^{14}$ which was originally developed as an anticancer agent. ${ }^{15}$ Recent studies have shown extra-telomeric functions of hTERT, including stabilizing mitochondria, promoting cellular proliferation, and antioxidant, anti-inflammatory, and anti-apoptotic effects. ${ }^{4,16}$ GV1001 may protect against IRI by mimicking these extratelomeric functions. In line with our finding that GV1001 showed protective effects against IRI in the brain, previous reports have described its protective effects against IRI in other organs. GV1001 significantly increased the survivability of skin flaps against IRI by reducing ROS levels and inflammation in a rat model. ${ }^{16}$ Furthermore, GV1001 showed potential protective effects in a myocardial IRI rat model, ${ }^{17}$ lung transplantation rat model, ${ }_{1}^{18}$ and renal IRI mouse model. ${ }^{14}$ However, to our knowledge, the present study is the first to show significant neuroprotective effects of GV1001 against cerebral IRI.

The PI3K pathway is important for cell survival and contributes to the protection of the brain and NSCs after ischemic injury. ${ }^{19}$ Activated PI3K phosphorylates Akt downstream, ${ }^{20}$ and pAkt inactivates glycogen GSK-3 $\beta$ by phosphorylation and inhibits caspase-9, which is related to apoptosis. ${ }^{21,22}$ Activated GSK-3 $\beta$ is associated with cell death due to ischemic injury. ${ }^{8}$ In the present study, the in vitro OGD/R model confirmed the neuroprotective mechanism of GV1001, which was achieved by increasing levels of the survival-related proteins (PI3K, pAkt, and pGSK-3 $\beta$ ) and decreasing levels of apoptosis-related proteins such as Bax, cytosolic cytochrome c, cleaved caspase-3, and cleaved caspase-9 (Figure 5A and B). These protective effects of GV1001 were significantly reduced by co-treatment with the PI3K inhibitor, LY294003 (Figure 6). In cortical neurons, GV1001 also increased the levels of survival-related proteins (IRS-1 and PI3K) and attenuated those of apoptosis-related proteins. We previously demonstrated the downstream PI3K pathway in cortical neurons after GV1001 treatment. ${ }^{5}$ These findings indicate the direct effect of GV1001 on the PI3K pathway. Similar to the results of the in vitro OGD/R model, the $\mathrm{PI} 3 \mathrm{~K}$ pathway was associated with the neuroprotective mechanisms of GV1001 in an ischemic stroke rat model. These neuroprotective mechanisms were achieved by increasing pAkt, pGSK-3 $\beta, p E R K 1 / 2$, and $\mathrm{Bcl}-2$ levels and by suppressing $B a x$ expression (Figure $2 \mathrm{~A}$ and $\mathrm{B}$ ), all of which are related to the PI3K pathway. ${ }^{21,23}$ Proteomics revealed that GV1001 increased the levels of diverse intracellular proteins, including elongation factor 1-delta, heat shock protein 90a, and ezrin in OGD/R-injured NCSs. These proteins can promote the PI3K pathway. ${ }^{24-26}$ Additionally, microarray data showed an increase in Akt expression with GV1001 treatment. After activation, NSCs differentiate into neural cells that replace damaged cells during 


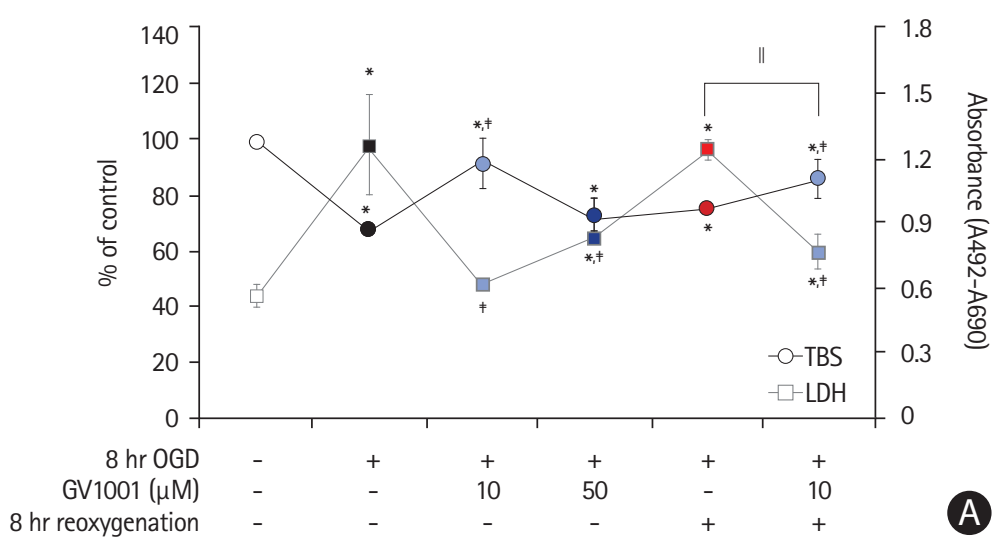

$8 \mathrm{hrOGD}$
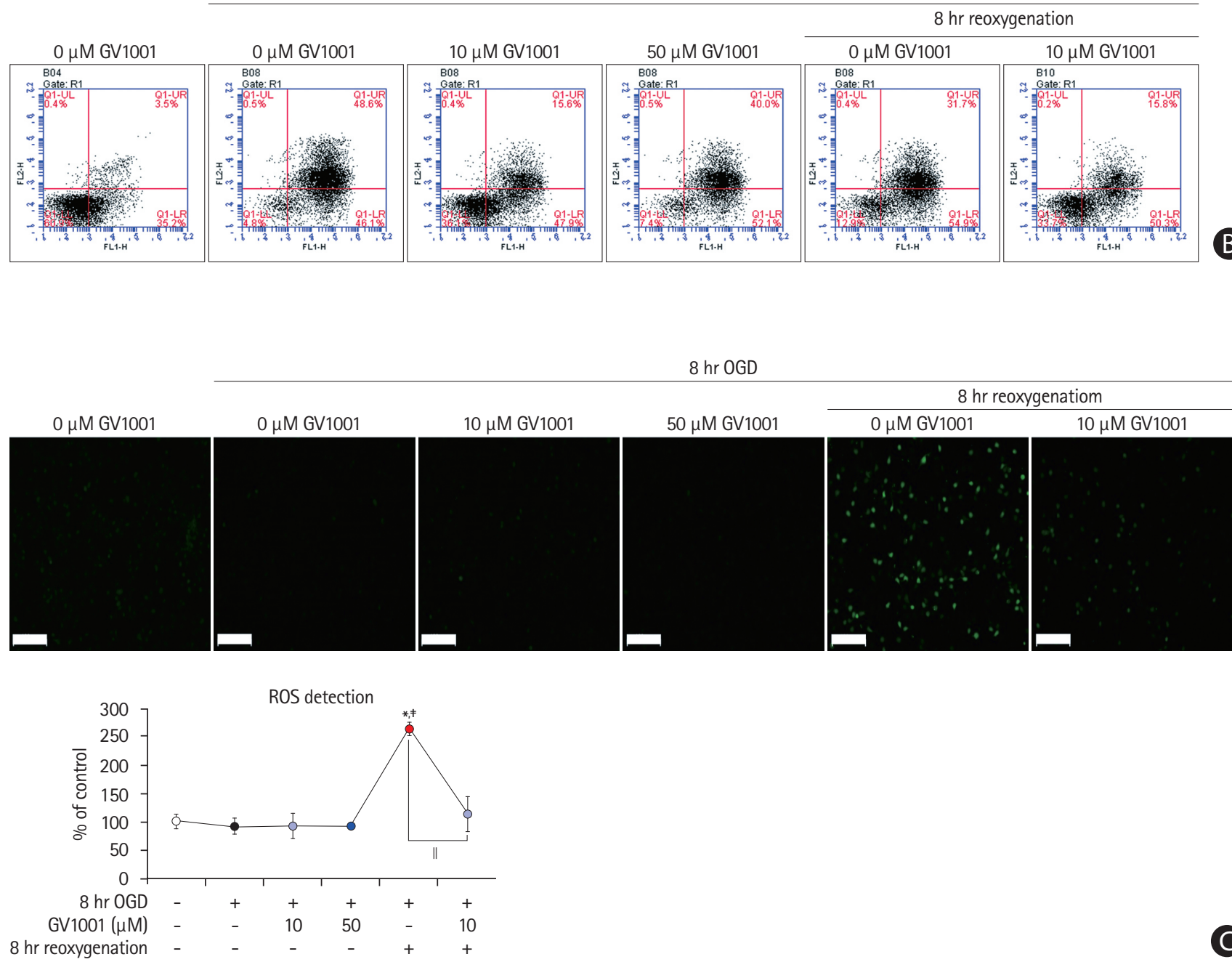

Figure 7. Effects of GV1001 on cortical neurons injured by oxygen-glucose deprivation/reoxygenation (OGD/R). GV1001 restored the viability (A) and alleviated the toxicity (B) of cortical neurons injured by OGD/R. (C) GV1001 significantly inhibited the production of reactive oxygen species (ROS), which was elevated by reoxygenation after OGD, in neurons, scale bar: $100 \mu \mathrm{m}$. (D) Treatment with GV1001 restored the migration activity of cortical neurons, which was reduced by 0GD/R. (E) OGD/R decreased the levels of survival-related proteins and increased those of death-associated proteins. Treatment with GV1001 reversed these changes. TBS, trypan blue staining; LDH, lactate dehydrogenase; IRS-1, insulin receptor substrate; PI3K, phosphoinositide 3-kinase; GAPDH, glyceraldehyde 3-phosphate dehydrogenase; Bax, Bcl-2 associated X. ${ }^{*} P<0.05$ (vs. control group); ${ }^{\dagger} P<0.01$ (vs. control group); ${ }^{*} P<0.05$ (vs. NSCs only treated with 0 GD); ${ }^{\circledR} P<0.01$ (vs. NSCs only treated with OGD); " $P<0.05$ (vs. NSCs treated with 0 GD/R). 

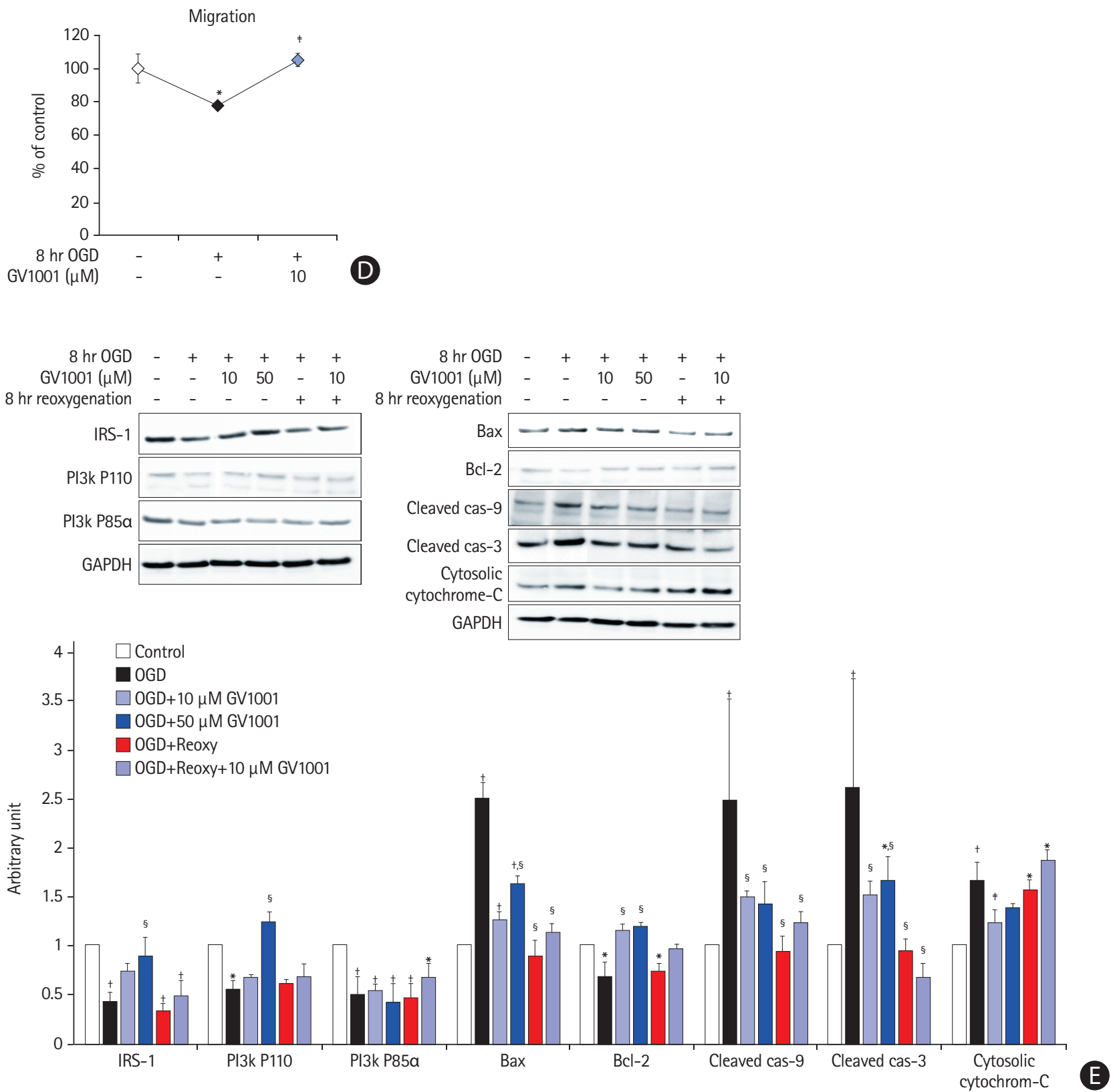

Figure 7. Continued.

neurogenesis. ${ }^{27}$ The replacement of cells by regeneration of endogenous NSCs might restore neurological function after ischemic stroke. ${ }^{27,28}$ However, endogenous NSCs are injured by ischemic stroke and lose their critical stem cell characteristics. SOX2 is a marker for multipotent NSCs. ${ }^{29}$ In the peri-infarct subventricular zone of the stroke rat model, SOX2 levels were markedly increased in the GV1001-treated groups compared with the saline group (Figure $2 \mathrm{C}$ ). An in vitro $0 \mathrm{GD} / \mathrm{R}$ model revealed that treatment with GV1001 restored the viability (Figure $3 \mathrm{C}$ ) and proliferation (Figure 3D and E) activity of OGD/Rinjured NSCs. GV1001 showed an anti-apoptotic effect (Figure $3 \mathrm{G}$ ) and recovered the impaired migratory activity (Figure $3 \mathrm{H}$ ).
In addition, GV1001 demonstrated an antioxidant effect by reducing ROS levels (Figure 4B) and decreasing oxidative mitochondrial damage caused by 0GD/R (Figure 4E). MDA and intracellular $\mathrm{Ca}^{2+}$ levels were assessed to verify the antioxidant effect of GV1001. GV1001 attenuated both MDA and intracellular $\mathrm{Ca}^{2+}$ levels, which were increased by $0 \mathrm{GD} / \mathrm{R}$ (Figure $4 \mathrm{C}$ and D).

The expressions of active $\beta$-catenin, cyclin D1, and c-myc were analyzed to evaluate the Wnt signaling pathway. This pathway has neuroprotective effects against and regenerative effects after ischemic injury. ${ }^{30}$ In this study, active $\beta$-catenin, cyclin D1, and c-myc levels were decreased in NSCs following 0GD/R injury 
but were restored by treatment with GV1001 (Figure 5C). These findings suggest that GV1001 has non-canonical telomerase activity, consistent with that seen in our previous study. ${ }^{5}$ Additionally, levels of Ki67, which reflects proliferative activity, ${ }_{1}^{31}$ and those of HMGB-1, which modulates DNA repair and chromatin modification after DNA damage, ${ }_{1}^{32}$ were reduced by $0 G D / R$, and treatment with GV1001 increased the levels of both (Figure 5A). Therefore, GV1001 may repair the DNA.

NSCs, which are activated under various conditions, differentiate into diverse types of nerve cells, including neurons, astrocytes, and oligodendrocytes. ${ }^{27}$ After a stroke, NSCs more frequently differentiate to form astrocytes, which contribute to gliosis, with less frequent differentiation to neurons. ${ }^{27}$ In this study, incremental increases in the levels of neuronal markers and decreases in the levels of astrocyte markers were evident in the peri-infarcted regions of the brains of GV1001-treated rates (Figure 2B). These findings can be explained by our previous report that GV1001 restores the ability of injured NSCs to differentiate more frequently to neurons and less frequently to

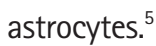

This study had two limitations. First, the pharmacokinetics of GV1001 were not evaluated. Second, no single animal model can reflect the heterogeneous nature of human stroke. Therefore, it is difficult to generalize the findings of the current study directly to ischemic stroke in humans.

\section{Conclusions}

In conclusion, we demonstrated the neuroprotective effect of GV1001 in an acute ischemic stroke animal model, as evidenced by improved tissue and neurological outcomes. GV1001 displayed neuroprotective effects against OGD/R injury in NSCs by inducing proliferation, restoring cell migration, reducing apoptosis, and stabilizing the mitochondria. These effects mimic the extra-telomeric functions of hTERT. Thus, GV1001 is a potential therapeutic candidate for ischemic stroke; however, further studies are needed to confirm this.

\section{Supplementary materials}

Supplementary materials related to this article can be found online at https://doi.org/10.5853/jos.2021.00626.

\section{Disclosure}

The authors have no financial conflicts of interest.

\section{Acknowledgments}

This research was supported by the Basic Science Research Program of the National Research Foundation of Korea, which is funded by the Ministry of Science, ICT, and Future Planning (2018R1D1A1A09082825 and 2018R1D1A1B07047722); a grant from the Korea Health Technology R\&D Project through the Korea Health Industry Development Institute (KHIDI) funded by the Ministry of Health \& Welfare, Republic of Korea (grant number: HI20C0253); and the Medical Research Center (2017R1A5A2015395).

\section{References}

1. Vidale $S$, Consoli A, Arnaboldi M, Consoli D. Postischemic Inflammation in Acute Stroke. J Clin Neurol 2017;13:1-9.

2. Jung JE, Kim GS, Chen H, Maier CM, Narasimhan P, Song YS, et al. Reperfusion and neurovascular dysfunction in stroke: from basic mechanisms to potential strategies for neuroprotection. Mol Neurobio/ 2010;41:172-179.

3. Park HH, Yu HJ, Kim S, Kim G, Choi NY, Lee EH, et al. Neural stem cells injured by oxidative stress can be rejuvenated by GV1001, a novel peptide, through scavenging free radicals and enhancing survival signals. Neurotoxicology 2016;55:131-141.

4. Martínez P, Blasco MA. Telomeric and extra-telomeric roles for telomerase and the telomere-binding proteins. Nat Rev Cancer 2011:11:161-176.

5. Park HH, Lee KY, Kim S, Lee JW, Choi NY, Lee EH, et al. Novel vaccine peptide GV1001 effectively blocks $\beta$-amyloid toxicity by mimicking the extra-telomeric functions of human telomerase reverse transcriptase. Neurobiol Aging 2014;35:12551274.

6. Ko YJ, Kwon KY, Kum KY, Lee WC, Baek SH, Kang MK, et al. The anti-inflammatory effect of human telomerase-derived peptide on P. gingivalis lipopolysaccharide-induced inflammatory cytokine production and its mechanism in human dental pulp cells. Mediators Inflamm 2015;2015:385127.

7. Koh SH, Park Y, Song CW, Kim JG, Kim K, Kim J, et al. The effect of PARP inhibitor on ischaemic cell death, its related inflammation and survival signals. Eur J Neurosci 2004;20:14611472.

8. Koh SH, Yoo AR, Chang DI, Hwang SJ, Kim SH. Inhibition of GSK-3 reduces infarct volume and improves neurobehavioral functions. Biochem Biophys Res Commun 2008;371:894-899.

9. Kim YS, Yoo A, Son JW, Kim HY, Lee YJ, Hwang S, et al. Early activation of phosphatidylinositol 3-kinase after ischemic stroke reduces infarct volume and improves long-term behavior. Mol Neurobiol 2017:54:5375-5384. 
10. Chen J, Li Y, Wang L, Zhang Z, Lu D, Lu M, et al. Therapeutic benefit of intravenous administration of bone marrow stromal cells after cerebral ischemia in rats. Stroke 2001;32: 1005-1011.

11. Sughrue ME, Mocco J, Komotar RJ, Mehra A, D'Ambrosio AL, Grobelny BT, et al. An improved test of neurological dysfunction following transient focal cerebral ischemia in rats. $J$ Neurosci Methods 2006;151:83-89.

12. Studer L, Tabar V, McKay RD. Transplantation of expanded mesencephalic precursors leads to recovery in parkinsonian rats. Nat Neurosci 1998;1:290-295.

13. Currle DS, Hu JS, Kolski-Andreaco A, Monuki ES. Culture of mouse neural stem cell precursors. J Vis Exp 2007;2:152.

14. Koo TY, Yan JJ, Yang J. Protective effect of peptide GV1001 against renal ischemia-reperfusion injury in mice. Transplant Proc 2014;46:1117-1122.

15. Kyte JA. Cancer vaccination with telomerase peptide GV1001. Expert Opin Investig Drugs 2009;18:687-694.

16. Lee YK, Nata'atmaja BS, Kim BH, Pak CS, Heo CY. Protective effect of telomerase-based 16-mer peptide vaccine (GV1001) on inferior epigastric island skin flap survivability in ischaemia-reperfusion injury rat model. J Plast Surg Hand Surg 2017;51:210216.

17. Chang JE, Kim HJ, Jheon S, Lim C. Protective effects of GV1001 on myocardial ischemia-reperfusion injury. Mol Med Rep 2017;16:7315-7320.

18. Chang JE, Kim HJ, Yi $E_{1}$ Jheon S, Kim K. Reduction of ischaemiareperfusion injury in a rat lung transplantation model by lowconcentration GV1001. Eur J Cardiothorac Surg 2016;50:972979.

19. Son JW, Choi H, Yoo A, Park HH, Kim YS, Lee KY, et al. Activation of the phosphatidylinositol 3-kinase pathway plays important roles in reduction of cerebral infarction by cilnidipine. J Neurochem 2015;135:186-193.

20. Pap $M$, Cooper GM. Role of translation initiation factor $2 B$ in control of cell survival by the phosphatidylinositol 3-kinase/ Akt/glycogen synthase kinase 3beta signaling pathway. Mol Cell Biol 2002;22:578-586.

21. Hemmings BA, Restuccia DF. The PI3K-PKB/Akt pathway.
Cold Spring Harb Perspect Biol 2015;7:a026609.

22. Song WJ, Song EA, Jung MS, Choi SH, Baik HH, Jin BK, et al. Phosphorylation and inactivation of glycogen synthase kinase $3 \beta$ (GSK3 $\beta$ ) by dual-specificity tyrosine phosphorylation-regulated kinase 1A (Dyrk1A). J Biol Chem 2015;290: 2321-2333.

23. Yamaguchi $H$, Wang $H G$. The protein kinase PKB/Akt regulates cell survival and apoptosis by inhibiting Bax conformational change. Oncogene 2001;20:7779-7786.

24. Xie C, Zhou M, Lin J, Wu Z, Ding S, Luo J, et al. EEF1D promotes glioma proliferation, migration, and invasion through EMT and PI3K/Akt pathway. Biomed Res Int 2020;2020: 7804706.

25. Hemmings BA, Restuccia DF. PI3K-PKB/Akt pathway. Cold Spring Harb Perspect Biol 2012;4:a011189.

26. Quan C, Sun J, Lin Z, Jin T, Dong B, Meng Z, et al. Ezrin promotes pancreatic cancer cell proliferation and invasion through activating the Akt/mTOR pathway and inducing YAP translocation. Cancer Manag Res 2019;11:6553-6566.

27. Koh SH, Park HH. Neurogenesis in stroke recovery. Trans/ Stroke Res 2017;8:3-13.

28. Zhang $S$, Lachance BB, Moiz B, Jia X. Optimizing stem cell therapy after ischemic brain injury. J Stroke 2020;22:286305.

29. Ellis $P$, Fagan BM, Magness ST, Hutton S, Taranova O, Hayashi $\mathrm{S}$, et al. SOX2, a persistent marker for multipotential neural stem cells derived from embryonic stem cells, the embryo or the adult. Dev Neurosci 2004;26:148-165.

30. Wei ZZ, Zhang JY, Taylor TM, Gu X, Zhao Y, Wei L. Neuroprotective and regenerative roles of intranasal Wnt-3a administration after focal ischemic stroke in mice. J Cereb Blood Flow Metab 2018;38:404-421.

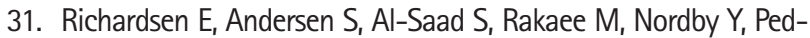
ersen $\mathrm{MI}$, et al. Evaluation of the proliferation marker Ki-67 in a large prostatectomy cohort. PLoS One 2017;12:e0186852.

32. Lange SS, Mitchell DL, Vasquez KM. High mobility group protein B1 enhances DNA repair and chromatin modification after DNA damage. Proc Natl Acad Sci U S A 2008;105: 10320-10325. 


\section{Supplementary methods}

\section{In vivo methods}

\section{Middle cerebral artery occlusion of rats}

After a 7-day adaptation and pre-training period for neurological examination, the rats were subjected to left middle cerebral artery occlusion (MCA0) for 2 hours using the intraluminal filament technique. The rats were anesthetized with isoflurane (3\% for induction and 2\% for surgical procedure) in a mixture of oxygen/nitrous oxide (30\%/70\%). Body temperature was maintained at $36.6^{\circ} \mathrm{C} \pm 0.5^{\circ} \mathrm{C}$ with a thermistor-controlled heating pad. Arterial $\mathrm{pH}_{1} \mathrm{pCO}_{2}, \mathrm{pO}_{2}$, and hematocrit were measured using a blood analysis system (International Technidyne, Edison, NJ, USA) in $0.1 \mathrm{~mL}$ of arterial blood obtained from a right femoral catheter. Arterial pressure was monitored from the femoral catheter with a strain-gauge transducer (LIFE KIT DX360, Nihon Kohden, Tokyo, Japan) and amplifier (MacLab Bridge Amplifier, AD Instruments Pty Ltd., Castle Hill, Australia). Phasic pressure, mean arterial pressure, and heart rate were recorded at a sampling rate of 200/seconds using a data acquisition system and a laboratory computer (MacLab 8 analog-todigital converter and Macintosh computer). After 2 hours of occlusion, reperfusion was performed.

For the sham surgery, a needle was introduced into the left common carotid artery of the rats and was immediately withdrawn. Regional cerebral blood flow was measured using a laser Doppler flowmeter (ALF21, Advance, Tokyo, Japan) and a wire-type probe (0.3 mm diameter; Unique Medical, Tokyo, Japan). These flowmeters and probes were inserted through a small burr hole $2 \mathrm{~mm}$ lateral to the bregma at the surface of the cortex to evaluate the ischemic core in the caudate and putamen.

\section{Transferase-mediated deoxyuridine triphosphate nick end labeling assay}

After sacrificing the rats, they were transcardially perfused with $0.9 \%$ sodium chloride. Brains were swiftly removed, fixed overnight in 4\% paraformaldehyde solution, and then cryoprotected in a 30\% sucrose solution for 3 days. Each brain was mounted on a cold metal block using an optimal cutting temperature compound (Sakura Tissue Tek, Torrance, CA, USA). Coronal sections (20 $\mu \mathrm{m}$ thickness) were cut using a motorized cryostat (Leica, Richmond, IL, USA) at $-20^{\circ} \mathrm{C}$. Slides were stored at $-4^{\circ} \mathrm{C}$ and processed for transferase-mediated deoxyuridine triphosphate nick end labeling (TUNEL) staining. After washing the fixed tissues with phosphate-buffered saline (PBS) for 30 minutes, the tissues were incubated with $3 \% \mathrm{H}_{2} \mathrm{O}_{2}$ in meth- anol for 10 minutes. The tissues were rinsed with PBS and placed in $0.1 \%$ Triton $X \unrhd 100$ in $0.1 \%$ sodium citrate for 2 minutes on ice. After washing the tissues three more times, the TUNEL reaction mixture was added, and the tissues were finally incubated at $37^{\circ} \mathrm{C}$ without light for 1 hour. After washing three times with PBS, the tissues were mounted with 4',6囚diamidino囚2区phenylindole (DAPI) mounting medium (Vector Laboratories, Burlingame, CA, USA), and the number of TUNEL-positive cells was counted.

\section{Histology and immunohistochemistry}

All rats used for the evaluation of the infarct volume with magnetic resonance imaging (MRI) were sacrificed 48 hours after MCAO for histological and immunohistochemistry analyses. The rats were transcardially perfused with PBS. The brains were quickly removed and cooled in ice-cold artificial cerebrospinal fluid for 5 minutes. Subsequently, $2 \mathrm{~mm}$ thick slices of the brain matrix were prepared by sectioning along the coronal plane approximately $0.4 \mathrm{~mm}$ from the bregma point on the skull. The slices were fixed overnight in $4 \%$ paraformaldehyde solution and then cryoprotected in a 30\% sucrose solution for 3 days. The remaining brain tissues were stored at $-80^{\circ} \mathrm{C}$ for Western blotting. Each brain specimen was mounted on a cold metal block using an optimal cutting temperature compound (Leica, Wetzlar, Germany), and coronal brain sections (20 $\mu \mathrm{m}$ in thickness) were cut on a motorized cryostat (Leica, Wetzlar, Germany) at $-20^{\circ} \mathrm{C}$. Three sections were placed on one "Muto" glass slide by pressing the slide onto the section as it came off the cryostat blade. Slides were stored at $-80^{\circ} \mathrm{C}$ and processed for hematoxylin and eosin (H\&E) and TUNEL staining. H\&E staining distinguished the peri-infarct region. TUNEL-positive cells were counted in both the GV1001 and control groups. Immunohistochemical staining was performed using antibodies against phosphorylated Akt (pAkt; Ser473; 1:100, 9271; Cell Signaling Technology, Beverly, MA, USA), phospho-glycogen synthase kinase (pGSK-3 $\beta, 1: 1,000,9336$, Cell Signaling Technology), phosphorylated-extracellular signal-regulated kinase (pERK1/2, Thr202/Tyr204; 1:1,000, 9101, Cell Signaling Technology), phosphorylated p38 (Thr180/182; 1:1,000, 9211, Cell Signaling Technology), B-cell lymphoma 2 (Bcl-2; 1:100, 2876, Cell Signaling Technology), nestin (1:200, ab6142, Abcam, Cambridge, MA, USA), neuronal nuclei (NeuN; 1:100; MAB377, Millipore, Bedford, MA, USA), and doublecortin (DCX; 1:100, ab28941, Abcam). Immunohistochemically positive cells were identified using a fluorescence microscope (Olympus, Tokyo, Japan). The number of positive cells in the peri-infarct region of the brain of the control group and in the corresponding area of the brain in the GV1001-treated group was expressed as the 
average number of positive cells per high-power field $(\times 200)$. As a negative control, the above procedures were repeated without a primary antibody; this sample showed no stained cells.

\section{Western blotting}

Frozen brain tissues of rats were rapidly micro-dissected on an ice-chilled plate. The peri-infarct region in the control group and the corresponding area in the GV1001-treated group, which were confirmed based on the MRI findings, were used in the Western blot analysis. The micro-dissected tissues were homogenized using a T 10 basic homogenizer (IKA Laboratory, Wilmington, NC, USA) and a type B pestle in 10:1 volume/ weight buffer containing RIPA II cell lysis buffer 1× EDTA-free Triton, $1 \mathrm{mM}$ phenylmethylsulfonyl fluoride (PMSF), $1 \mathrm{mM}$ sodium fluoride, $1 \mathrm{mM}$ sodium orthovanadate $\left(\mathrm{Na}_{3} \mathrm{VO}_{4}\right)$, and $0.5 \% 1 \times$ protease inhibitor cocktail. The protein concentrations of the tissue lysates were determined using a protein assay kit (Bio-Rad, Hercules, CA, USA). Equal amounts $(20 \mu \mathrm{g})$ of protein were resolved by $10 \%$ sodium dodecyl sulfate-polyacrylamide gel electrophoresis and transferred to nitrocellulose membranes (Amersham Pharmacia Biotech, Piscataway, NJ, USA). The membranes were blocked with 5\% skim milk and sequentially incubated with the same antibodies against phospho-insulin receptor substrate-1 (pIRS-1) versus neural stem cells (NSCs) only treated with OGD (Ser636/639) (1:1,000, 2388, Cell Signaling Technology), pAkt (ser473; 1:500, 9271, Cell Signaling Technology), Akt (1:2,000, 9272, Cell Signaling Technology), pGSK-3 $\beta$ (1:1,000, 9336, Cell Signaling Technology), GSK-3ß (1:2,000, sc-9166, Santa Cruz Biotechnology, Dallas, TX, USA), pERK1/2 (Thr202/Tyr204; 1:1,000, 9101, Cell Signaling Technology), phosphorylated p38 (Thr180/182; 1:1,000, 9211, Cell Signaling Technology), Bcl-2 (1:1,000, 2876, Cell Signaling Technology), Bcl-2 associated X (Bax; 1:1,000, 2772, Cell Signaling Technology), and $\beta$-tubulin $(1: 2,000,2146$, Cell Signaling Technology). The membranes were washed with Trisbuffered saline containing 0.1\% Tween-20 (TBST) and processed using horseradish peroxidase-conjugated anti-rabbit antibody (Jackson ImmunoResearch Laboratories Inc., West Grove, PA, USA), followed by enhanced chemiluminescence detection (GenDEPOT, Katy, TX, USA). The Western blot results were quantified using an image analyzer (ImageQuant LAS 4000, GE Healthcare, Little Chalfont, UK).

\section{In vitro methods}

\section{Culturing of neural stem cells}

Rat embryos were decapitated on embryonic day 13 . Their brains were rapidly removed and placed in a Petri dish half-full of ice-cold Hank's balanced salt solution (137 mM NaCl, 5.4 $\mathrm{mM} \mathrm{KCl}, 0.3 \mathrm{mM} \mathrm{Na}_{2} \mathrm{HPO}_{4}, 0.4 \mathrm{mM} \mathrm{KH}_{2} \mathrm{PO}_{4}, 5.6 \mathrm{mM}$ glucose, and 2.5 mM HEPES; GIBCO BRL, Carlsbad, CA, USA). Single cells were dissociated from the whole cerebral cortex, lateral ganglionic eminence, and ventral midbrain of rat embryos. The resulting cells were plated at a density of $2 \times 10^{4} \mathrm{cell} / \mathrm{s} / \mathrm{cm}^{2}$ in culture dishes pre-coated with poly-L-ornithine/fibronectin in $\mathrm{Ca}^{2+} / \mathrm{Mg}^{2+}$-free PBS (GIBCO BRL) and cultured in N2 medium (Dulbecco's modified Eagle's medium [DMEM]/nutrient mixture F-12, 25 mg/L insulin, $100 \mathrm{mg} / \mathrm{L}$ transferrin, $30 \mathrm{nM}$ selenite, $100 \mu \mathrm{M}$ putrescine, $20 \mathrm{nM}$ progesterone, $0.2 \mathrm{mM}$ ascorbic acid, $2 \mathrm{nM} \mathrm{L-glutamine,} 8.6 \mathrm{mM} \mathrm{D}[+]$ glucose, and $20 \mathrm{nM} \mathrm{NaHCO}_{3}$; Sigma-Aldrich, St. Louis, MO, USA) supplemented with basic fibroblast growth factor ( $10 \mathrm{ng} / \mathrm{mL}$, R\&D Systems, Minneapolis, $\mathrm{MN}$, USA). Cultures were maintained at $37^{\circ} \mathrm{C}$ in a humidified $5 \% \mathrm{CO}_{2}$ atmosphere for 4 to 6 days.

\section{Culturing of primary cortical neurons}

Primary cultured cortical neurons were obtained from the cerebral cortex of fetal Sprague-Dawley rats (Orient Bio, Seongnam, Korea) on E16. The embryos were decapitated, and the brains were rapidly removed and placed in a Petri dish halffilled with ice-cold Hank's balanced salt solution $(137 \mathrm{mM}$ $\mathrm{NaCl}, 5.4 \mathrm{mM} \mathrm{KCl}, 0.3 \mathrm{mM} \mathrm{Na}_{2} \mathrm{HPO}_{4}, 0.4 \mathrm{mM} \mathrm{KH}_{2} \mathrm{PO}_{4}, 5.6 \mathrm{mM}$ glucose, and $2.5 \mathrm{mM}$ HEPES; GIBCO BRL). Single cells dissociated from the whole cerebral cortex of the rat embryos were plated on a poly-L-lysine (Sigma-Aldrich) pre-coated $100 \mathrm{~mm}$ Corning dish $\left(5 \times 10^{6}\right.$ cells $\left./ \mathrm{cm}^{2}\right)$ or glass coverslips placed in 6or 24-well plates (Nunc, Roskilde, Denmark) at a cell density of $5 \times 10^{5}$ or $2.5 \times 10^{6} \mathrm{cell} / \mathrm{s} / \mathrm{cm}^{2}$. Cultures were incubated in DMEM (high concentration of glucose) supplemented with $10 \%$ heatinactivated fetal bovine serum (1.7 days after plating), 1\% penicillin-streptomycin, $3.7 \mathrm{~g} / \mathrm{L} \mathrm{NaHCO}_{3}, 0.5 \mu \mathrm{g} / \mathrm{mL}$ insulin, and p-aminobenzoic acid. Cultures were maintained at $37^{\circ} \mathrm{C}$ in a humidified atmosphere containing $5 \% \mathrm{CO}_{2}$. Two days after plating, non-neuronal cells were removed by adding $5 \mu \mathrm{M}$ cytosine arabinoside for 24 hours. Only mature cultures ( 7 days in vitro) were used for the experiments. The population of neuronal cells was approximately $79.7 \%$ in cultured primary cortical neurons.

Trypan blue staining and lactate dehydrogenase release assay

Trypan blue staining and lactate dehydrogenase release assay were performed to assess cell viability and cytotoxicity, respectively. For trypan blue staining, $10 \mu \mathrm{L}$ samples of cells were incubated with $10 \mu \mathrm{L}$ of trypan blue solution for 2 minutes. Un- 
stained live cells were counted using a hemocytometer. A colorimetric assay kit (Roche Boehringer Mannheim, Indianapolis, IN, USA) was used to quantify the amount of lactate dehydrogenase released from cultured NSCs according to the manufacturer's instructions. Cell viability was assessed using a Synergy $\mathrm{H} 1$ Hybrid enzyme-linked immunosorbent assay (ELISA) plate reader (BioTek Instruments, Winooski, VT, USA) by measuring the absorbance at $490 \mathrm{~nm}$ at a reference wavelength of $690 \mathrm{~nm}$. All results were normalized to the optical density (OD) of an identical well without cells.

\section{Bromodeoxyuridine cell proliferation assay}

NSCs were incubated in bromodeoxyuridine (BrdU) labeling medium (10 $\mu \mathrm{M}$ BrdU) for 5 hours. Cell proliferation was measured using a BrdU Labeling and Detection Kit (Roche Boehringer Mannheim, Grenzach-Wyhlen, Germany) according to the manufacturer's instructions. Cell proliferation was assessed by measuring the absorbance of each well at $370 \mathrm{~nm}$ (reference wavelength, $492 \mathrm{~nm}$ ) using a Synergy $\mathrm{H} 1$ spectrophotometer plate reader (BioTek Instruments). All results were adjusted by subtracting the $\mathrm{OD}$ of an identical well without cells.

\section{Colony-forming unit assay}

The proliferation of NSCs was assessed using a colony-forming unit assay. Approximately $0.5 \times 10^{4}$ cells were seeded in a 60 $\mathrm{mm}$ grid plate and treated with oxygen-glucose deprivation/ reoxygenation (OGD/R) and GV1001 (10 and $50 \mu \mathrm{M})$ for 8 hours. The cells were washed with Dulbecco's PBS (DPBS) and incubated with a fresh culture medium. After 14 days, the cells were washed again with DPBS and stained with $0.5 \%$ crystal violet (Sigma-Aldrich) in methanol for 30 minutes at room temperature. After staining, the plates were washed with DPBS and allowed to dry. Colonies were counted using a dissecting microscope. Colonies $<2 \mathrm{~mm}$ in diameter or those that were faintly stained were excluded.

\section{Annexin V/propidium iodide apoptosis assay}

Apoptosis was measured using the fluorescein isothiocyanate (FITC) Annexin V Apoptosis Detection Kit I (Beckton Dickinson, Franklin Lakes, NJ, USA) according to the manufacturer's instructions. Briefly, NSCs were exposed to OGD with GV1001 (10 and $50 \mu \mathrm{M}$ ) and incubated for 8 hours at $37^{\circ} \mathrm{C}$. Thereafter, the cells were rinsed twice with cold PBS and then resuspended in $1 \times$ binding buffer. Cells were then transferred $(100 \mu \mathrm{L}$ of the solution $\left[1 \times 10^{5}\right.$ cells]) to a $1.5 \mathrm{~mL}$ culture tube followed by application of $5 \mu \mathrm{L}$ of FITC Annexin $\mathrm{V}$ and $10 \mu \mathrm{L}$ propidium iodide. Samples were gently mixed and incubated for 15 minutes at room temperature in the dark. Finally, $400 \mu \mathrm{L}$ of $1 \times$ binding buffer was added to each tube, and samples were analyzed using flow cytometry (Accuri C6 Flow cytometer, BD Biosciences, San Jose, CA, USA). Data were acquired and analyzed using BD Accuri C6 software.

\section{Evaluation of apoptosis}

NSCs were seeded on a 4-well chamber slide containing removable wells (Thermo Fisher Scientific, Waltham, MA, USA). The cells were exposed to OGD and GV1001 (10 or $50 \mu \mathrm{M}$ ) or OGD/R (exposed to OGD for 8 hours and subsequently reoxygenated for 8 hours) and $10 \mu \mathrm{M}$ GV1001. The cells were then rinsed twice with PBS, air-dried, and fixed with 4\% paraformaldehyde in PBS for 1 hour at room temperature. Apoptotic cell death was assessed using the TUNEL labeling assay (Roche Boehringer Mannheim, Indianapolis, IN, USA). To monitor intact, condensed, or fragmented nuclei, TUNEL-stained cells were counterstained with DAPI (Vector Laboratories, Burlingame, CA, USA) for 20 minutes, washed several times with PBS, and mounted on glass slides with mounting medium (Merck, Kenilworth, NJ, USA). The cells were then observed under a Bx53 microscope (Olympus).

\section{Migration assay}

The assay was performed using a OCM 24-well colorimetric cell migration assay kit (Chemicon, Temecula, CA, USA) according to the manufacturer's instructions. NSCs $\left(3 \times 10^{5}\right.$ cells $\left./ \mathrm{mL}\right)$ treated with $0 \mathrm{GD}$ and GV1001 (10 or $50 \mu \mathrm{M}$ ) or $\mathrm{OGD} / \mathrm{R}$ and 10 $\mu \mathrm{M}$ GV1001 were added to the upper chamber of the plate and incubated for 24 hours at $37^{\circ} \mathrm{C}$. The cells that migrated through the membrane were stained and enumerated by measuring the absorbance at $560 \mathrm{~nm}$ using a Synergy $\mathrm{H} 1$ spectrophotometer plate reader (BioTek Instruments), as previously described. ${ }^{16}$

\section{Determination of free radical production}

Cells were exposed to OGD and GV1001 (10 or $50 \mu \mathrm{M}$ ) or OGD/ $\mathrm{R}$ and $10 \mu \mathrm{M}$ GV1001 and incubated with the fluorescent probe $2 ', 7^{\prime}$-dichlorodihydrofluorescein diacetate $\left(\mathrm{H}_{2} \mathrm{DCF}-\mathrm{DA}\right.$; Molecular Probes, Eugene, OR, USA) for 15 minutes to measure free radical production. After incubation with $10 \mu \mathrm{M} \mathrm{H} \mathrm{H}_{2}$ DCF$\mathrm{DA}$ at $37^{\circ} \mathrm{C}$ for 15 minutes, the cells were washed three times with PBS. In the assay, $\mathrm{H}_{2} \mathrm{DCF}-\mathrm{DA}$ freely crosses cell membranes and is hydrolyzed by cellular esterase to $\mathrm{H}_{2} \mathrm{DCF}$, which is subsequently oxidized to the fluorescent molecule 2'7'-dichlorofluorescein (DCF) in the presence of peroxides. Therefore, DCF fluorescence is indicative of the level of intracellular hydrogen peroxide, but not of superoxide levels. The intracellular accumulation of DCF was assessed by measuring the fluorescence (exci- 
tation at $488 \mathrm{~nm}$; emission at $530 \mathrm{~nm}$ ) of each well using a Synergy H1 spectrophotometer plate reader (BioTek Instruments). All results were adjusted by subtracting the $\mathrm{OD}$ of an identical well without cells.

\section{Malondialdehyde assay of lipid peroxidation}

The assay was performed using a lipid peroxidation (malondialdehyde [MDA]) assay kit (Sigma-Aldrich) according to the manufacturer's instructions. In brief, cells $\left(1 \times 10^{6} / \mathrm{mL}\right)$ were homogenized on ice in $300 \mu \mathrm{L}$ of MDA lysis buffer containing 3 $\mu \mathrm{L}$ of butylated hydroxytoluene. The cells were then incubated with $600 \mu \mathrm{L}$ of thiobarbituric acid solution at $95^{\circ} \mathrm{C}$ for 1 hour. The MDA level of each sample was assessed by measuring the absorbance at $532 \mathrm{~nm}$ using a Synergy $\mathrm{H} 1$ spectrophotometer plate reader (BioTek Instruments).

\section{Determination of $\mathrm{Ca}^{2+}$ level}

NSCs were treated for 8 hours, and the culture medium was replaced with a fresh medium containing $5 \mu \mathrm{M}$ fluo-4 acetoxymethyl (AM) (Life Technologies, Carlsbad, IL, USA). The cells were then incubated at $37^{\circ} \mathrm{C}$ for 1 hour. The cells were washed in PBS and incubated for another 30 minutes to allow complete de-esterification of intracellular AM esters for fluorescence analysis. The accumulation of fluo-4 AM in the cells was assessed using a Synergy H1 ELISA plate reader (BioTek Instruments) by measuring the fluorescence (excitation $505 \mathrm{~nm}$; emission $530 \mathrm{~nm}$ ). Fluo-4 AM intensity was quantified within a region of interest for each cell and was expressed as the relative change in fluorescence.

$\Delta F \mid F_{0}=\left(F-F_{0}\right) / F_{0}$

Where $F_{0}$ is the fluorescence level at the start of the experiment, after subtracting the background fluorescence. Peak amplitudes of $\Delta F \mid F_{0}$ were determined after stimulation. Cumulative increases in $\triangle F \mid F_{0}$ were calculated for 420 seconds following stimulation using Excel software (Microsoft, Redmond, WA USA).

\section{Oxidative mitochondrial DNA damage assay}

Oxidative mitochondrial DNA damage was measured using an OxiSelect Oxidative DNA Damage ELISA kit for 8-hydroxy-deoxyguanine (8-OHdG; Cell Biolabs, San Diego, CA, USA) according to the manufacturer's instructions. Mitochondrial DNA was extracted using the Purelink Genomic DNA Mini Kit (Invitrogen, Carlsbad, CA, USA). Samples/standards were added to the pre-adsorbed $8-\mathrm{OHdG} /$ bovine serum albumin conjugate. Subsequently, an anti-8-OHdG monoclonal antibody was added, followed by a horseradish peroxidase-conjugated secondary antibody, and quantified with authentic 8-OHdG
(Cell Biolabs) as a standard. Measurements were performed using a Synergy H1 ELISA plate reader (BioTek Instruments) with absorbance at $450 \mathrm{~nm}$.

\section{Adenosine triphosphate assay}

Adenosine triphosphate (ATP) concentrations were measured using an ATP assay kit (Abcam) according to the manufacturer's instructions. The fluorometric assessment was performed using a Synergy H1 ELISA plate reader (BioTek Instruments). ATP concentrations were detected at fluorescence excitation and emission wavelengths of 535 and $587 \mathrm{~nm}$, respectively.

\section{Mitochondrial membrane potential assay}

NSCs were seeded at a density of $5 \times 10^{5} / \mathrm{mL}$ in a 96-well culture plate. The cells were exposed to OGD and GV1001 $(1,10$, or $50 \mu \mathrm{M}$ ) or $\mathrm{OGD} / \mathrm{R}$ with $10 \mu \mathrm{M}$ GV1001 and incubated for 8 hours at $37^{\circ} \mathrm{C}$.

Mitochondrial membrane potential was measured using the JC-1 Mitochondrial Membrane Potential Assay Kit (Abnova, Taipei, Taiwan) according to the manufacturer's instructions using a Synergy H1 ELISA plate reader (BioTek Instruments). Healthy cells mainly comprising JC- $1 \mathrm{~J}$-aggregates were detected with fluorescence settings usually designed to detect rhodamine (excitation/emission wavelength $=540 / 570 \mathrm{~nm}$ ) or Texas Red (excitation/emission wavelength $=590 / 610 \mathrm{~nm}$ ). Apoptotic or unhealthy cells that mainly comprised JC-1 monomers were detected with settings designed to detect FITC (excitation/emission wavelength $=485 / 535 \mathrm{~nm}$ ).

\section{Western blot analysis}

The levels of several intracellular signaling proteins, including Ki67, p85a phosphoinositide 3-kinase (PI3K), pAkt (Ser 473), Akt, pGSK-3 $\beta$ (Ser 9), GSK-3 $\beta$, high mobility group box protein 1 (HMGB-1), Bax, cytosolic cytochrome c, procaspase-9, cleaved caspase-3 (Asp 175), phospho- $\beta$-catenin (Ser33/37/Thr41), nonphospho (active) $\beta$-catenin (Ser33/37/Thr41), c-Myc, cyclin $D 1$, and beta-tubulin ( $\beta$-tubulin), were analyzed by Western blotting immediately after 24 hours of treatment. Cells $\left(5 \times 10^{6}\right)$ were washed twice in cold PBS and incubated for 30 minutes on ice in lysis buffer (RIPA II cell lysis buffer $1 \times$ with Triton, without EDTA, $1 \mathrm{mM}$ PMSF, $1 \mathrm{mM}$ sodium fluoride, 1 $\mathrm{mM} \mathrm{Na}_{3} \mathrm{VO}_{4}$, and $0.5 \% 1 \times$ protease inhibitor cocktail). To evaluate cytosolic cytochrome c levels, cytosolic fractions were isolated using the mitochondria/cytosol fractionation kit (Abcam) according to the manufacturer's instructions. Briefly, after OGD for 8 hours and exposure to different concentrations of GV1001, NSCs were harvested, washed once with icecold PBS, and resuspended in $1.0 \mathrm{~mL}$ of $1 \times$ cytosol extraction 
buffer mix containing dithiothreitol (DTT) and protease inhibitors. After incubation on ice for 10 minutes, the cell suspension was sonicated using a Sonoplus apparatus 5 to 10 times on ice. The samples were centrifuged at $3,000 \mathrm{rpm}$ at $4^{\circ} \mathrm{C}$ for 10 minutes. The supernatants were centrifuged again at $13,000 \mathrm{rpm}$ for 30 minutes to separate the mitochondrial fraction (pellets) and the cytosolic fraction (supernatants). The mitochondrial pellet was washed once with the isolation buffer and then lysed in mitochondrial extraction buffer containing DT and protease inhibitors. Samples containing equal amounts (30 $\mu \mathrm{g}$ ) of protein were resolved by $4 \%$ to $12 \%$ sodium dodecyl sulfate-polyacrylamide gel electrophoresis (SDSPAGE). The resolved proteins were transferred onto nitrocellulose membranes (Amersham Pharmacia Biotech). The membranes were blocked with 2\% skim milk and incubated with the following specific primary antibodies: anti-Ki67 (1:100, ab16667, Abcam), phospho-phosphoinositide 3-kinase (pPI3K) p85(Tyr458)/p55(Tyr199) (p85a PI3K; 1:1,000, 4228, Cell Signaling Technology), pAkt (ser473; 1:500, 9271, Cell Signaling Technology), Akt (1:2,000, 9272, Cell Signaling Technology), pGSK-3 $\beta$ (1:1,000, 9336, Cell Signaling Technology), GSK-3 $\beta$ (1:2,000, sc-9166, Santa Cruz Biotechnology), HMGB-1 (1:1,000, 3935, Cell Signaling Technology), Bax (1:1,000, 2772, Cell Signaling Technology), cytochrome c (1:500, 4272, Cell Signaling Technology), caspase-9 (1:500, 9506, Cell Signaling Technology), cleaved caspase-3 (Asp175; 1:500, 9661, Cell Signaling Technology), phospho- $\beta$-catenin (Ser33/37/Thr41; 1:1,000, 9561, Cell Signaling Technology), non-phospho (active) $\beta$-catenin (Ser33/37/Thr41; 1:1,000, 8814, Cell Signaling Technology), c-Myc (1:1,000, 5605, Cell Signaling Technology), cyclin D1 $(1: 2,000,2926$, Cell Signaling Technology), and $\beta$-tubulin $(1: 2,000,2146$, Cell Signaling Technology). The membranes were washed with TBST and incubated with the appropriate horseradish peroxidase-conjugated anti-rabbit antibody (Jackson ImmunoResearch Laboratories Inc.), followed by enhanced chemiluminescence detection (GenDEPOT). Band intensities were quantified using an image analyzer (ImageQuant LAS 4000, GE Healthcare, Buckinghamshire, UK).

\section{Proteomics}

\section{1) Protein sample preparation}

Cells were washed twice with ice-cold PBS and sonicated for 10 seconds with a Sonoplus (Bandelin Electronics, Berlin, Germany) in a sample lysis solution composed of $7 \mathrm{M}$ urea and $2 \mathrm{M}$ thiourea with 4\% (w/v) 3-[(3-cholamidopropyl) dimethylammonio]1-propanesulfonate, 1\% (w/v) DT, 2\% (v/v) pharmalyte, and 1 $\mathrm{mM}$ benzamidine. Proteins were extracted for 1 hour at room temperature by vortexing. After centrifugation at $15,000 \times \mathrm{xg}$ for 1 hour at $15^{\circ} \mathrm{C}$, the insoluble material was discarded, and the soluble fraction was used for two-dimensional (2D) gel electrophoresis. Protein concentrations were determined using the Bradford method.

\section{2) 2D PAGE}

Immobiline DryStrip gel (IPG) dry strips (4 to $10 \mathrm{NL} I \mathrm{PG}, 13 \mathrm{~cm}$, GE Healthcare) were re-swollen for 9.5 hours with Destreak rehydration solution and 0.5\% IPG buffer and loaded with 150 $\mu \mathrm{g}$ of the sample. Isoelectric focusing (IEF) was performed at $20^{\circ} \mathrm{C}$ using an Ettan IPGphor 3 (GE Healthcare) following the manufacturer's instructions. For IEF, the voltage was linearly increased from 100 to $8,000 \mathrm{~V}$ over 7 hours for sample entry, followed by maintenance at a constant $8,000 \mathrm{~V}$. Focusing was complete after $55 \mathrm{kVh}$. Before the second dimension separation, the strips were incubated for 15 minutes in equilibration buffer (75 mM Tris-Cl, pH 8.8, containing $6 \mathrm{M}$ urea, 2\% SDS, $0.002 \% 1 \%$ bromophenol blue stock solution, and $29.3 \%$ glycerol), first with $1 \% \mathrm{DT}$ and then with $2.5 \%$ iodoacetamide. Equilibrated strips were inserted into sodium dodecyl sulfatepolyacrylamide gel electrophoresis (SDS-PAGE) gels $(13 \times 18$ $\mathrm{cm}, 12 \%)$, and SDS-PAGE was performed using a SE600 2D system (GE Healthcare) following the manufacturer's instructions. The $2 \mathrm{D}$ gels were run at $20^{\circ} \mathrm{C}$ for $1,700 \mathrm{Vh}$.

\section{3) Image analysis}

Quantitative analysis of digitized images was performed using ImageMaster 2D Platinum 7.0 software (GE Healthcare) according to the manufacturer's protocols. The intensity of each spot was normalized to the total valid spot intensity. The protein spots selected showed at least a two-fold difference in expression compared to those of the control or normal samples.

\section{4) Peptide mass fingerprinting}

For protein identification by peptide mass fingerprinting (PMF), protein spots were excised, digested with trypsin (Promega, Madison, WI, USA), and mixed with a cyano-4-hydroxycinnamic acid in 50\% acetonitrile/0.1\% trifluoroacetic acid. The samples were analyzed by matrix-assisted laser desorption ionization-time of flight mass spectrometry using a Microflex LRF 20 device (Bruker Daltonics, Billerica, MA, USA). Spectra were collected from 300 shots per spectrum over an $\mathrm{m} / \mathrm{z}$ range of 600 to 3,000 and were calibrated by a two-point internal calibration using trypsin auto-digestion peaks (m/z 842.5099, 2211.1046). The peak list was generated using Flex Analysis 3.0 software. The threshold used for peak selection was 500 for a minimum resolution of monoisotopic mass and 5 for signal-to- 
noise. The Mascot search program developed by Matrix Science (http://www.matrixscience.com/) was used for protein identification by PMF. The following parameters were used for the database search: trypsin as the cleaving enzyme, a maximum of one missed cleavage, iodoacetamide (Cys) as a complete modification, oxidation (Met) as a partial modification, monoisotopic mass, and a mass tolerance of $\pm 0.1 \mathrm{Da}$. The PMF acceptance criteria were based on probability scoring.

\section{Antibody microarray}

The antibody microarray analysis was performed using a Panorama Antibody Microarray-Cell Signaling kit (Sigma-Aldrich) according to the manufacturer's instructions. Briefly, $1.5 \times 10^{7}$ cells were seeded in $100 \mathrm{~mm}$ dishes and grown in the presence of OGD with GV1001 $(1,10$, or $50 \mu \mathrm{M})$ or $0 G D / R$ with $10 \mu \mathrm{M}$ GV1001 for 8 hours. Cells were collected, and protein samples were prepared according to the manufacturer's protocol. The protein samples were labeled with Cy3 or Cy5 (Amersham Biosciences) and subjected to antibody microarray analysis (Sigma-Aldrich). The array slides were scanned using a GenePix Personal 4100A scanner (Molecular Devices, San Jose, CA, USA), and the data were analyzed using GenePix Pro 5.0 (Molecular Devices). 

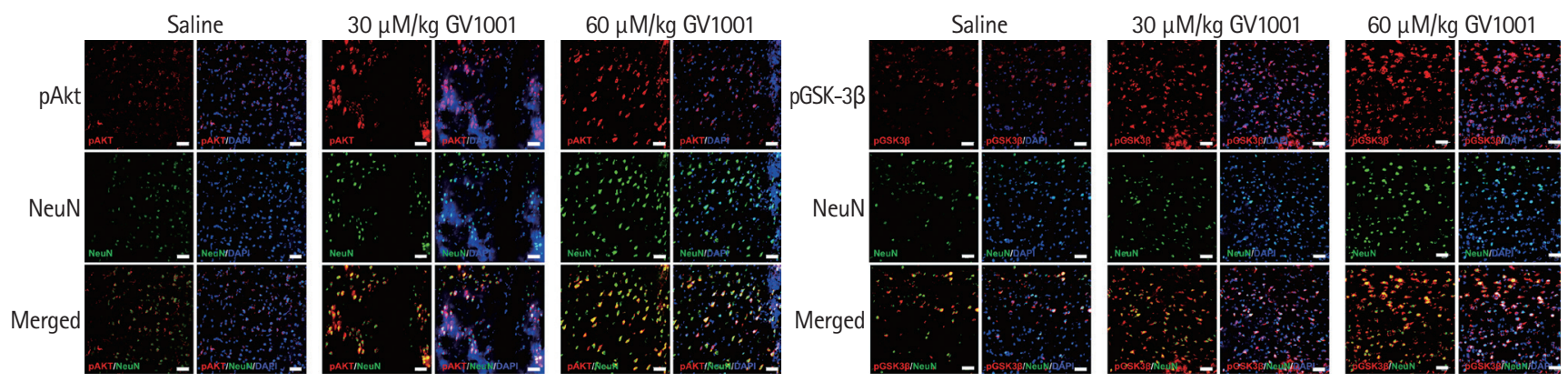

A

B

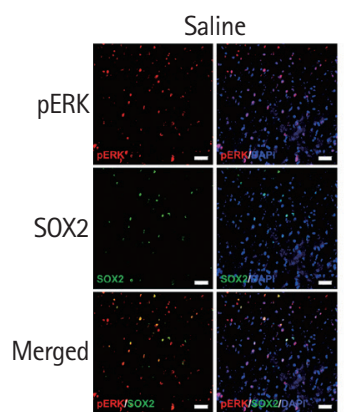

$30 \mu \mathrm{M} / \mathrm{kg}$ GV1001
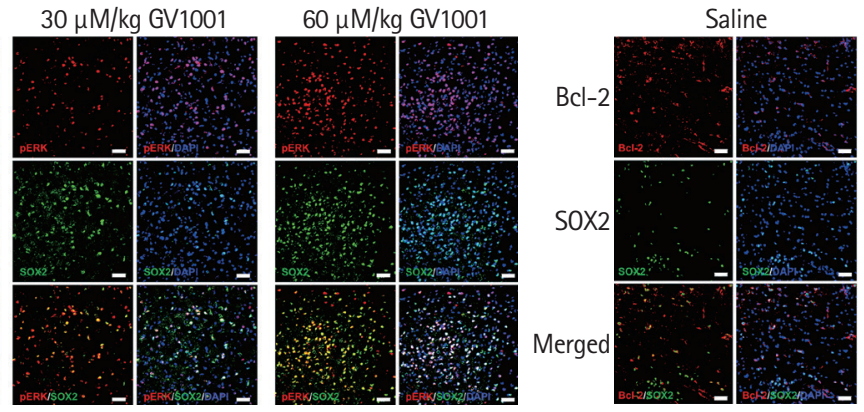

$30 \mu \mathrm{M} / \mathrm{kg}$ GV1001

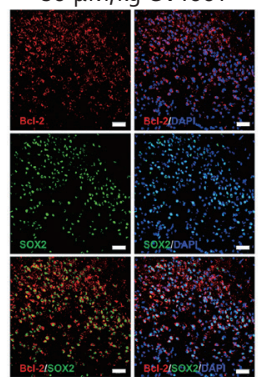

$60 \mu \mathrm{M} / \mathrm{kg}$ GV1001

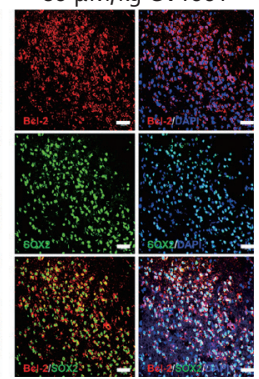

C

D

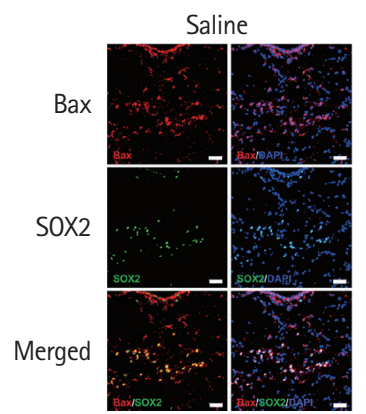

$30 \mu \mathrm{M} / \mathrm{kg}$ GV1001

$60 \mu \mathrm{M} / \mathrm{kg}$ GV1001
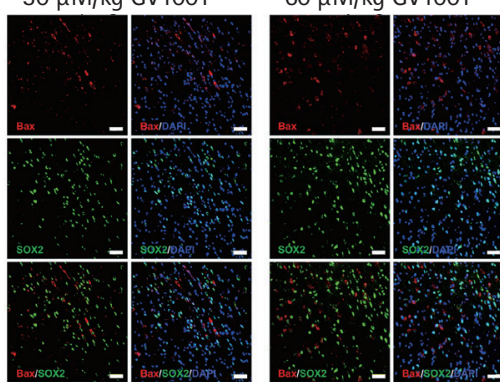

E

Supplementary Figure 1. Immunohistochemistry analysis showing the effect of GV1001 on the expression of intracellular signaling proteins, a neuronal nuclear marker (neuronal nuclei [NeuN])-positive cells, and SRY-box transcription factor 2 (SOX2; a neural stem cell marker)-positive cells in per-infarct regions of rats. In GV1001-treated groups, phosphorylated Akt (pAkt; Ser473) (A) and phospho-glycogen synthase kinase (pGSK-3 $\beta$; Ser9) (B) levels increased along with an increase in the number of NeuN-positive cells. Expression of phosphorylated-extracellular signal-regulated kinase (pERK) (C) and B-cell lymphoma 2 (Bcl-2) (D) increased and that of Bcl-2 associated X (Bax) (E) decreased with an increase in the number of SOX2-positive cells after GV1001 treatment, scale bar: $50 \mu \mathrm{m}$. 